\title{
An Intelligent Parallel Method for Automatic Production of the Human Bronchial Tree Fulfilling Bronchopulmonary Segmental Isolation Based on Stochastic Parametric L-system
}

\section{Zeinab Abbasi}

Sharif University of Technology

Ramin Bozorgmehry Boozarjomehry ( $\nabla$ rbozorgmehry@sharif.edu )

Sharif University Of Technology https://orcid.org/0000-0002-3270-9896

Research article

Keywords: Lung, Bronchial tree, L-System, conducting airways

Posted Date: May 26th, 2020

DOl: https://doi.org/10.21203/rs.3.rs-29636/v1

License: (c) (1) This work is licensed under a Creative Commons Attribution 4.0 International License. Read Full License 


\title{
An Intelligent Parallel Method for Automatic Production of the Human Bronchial Tree Fulfilling Bronchopulmonary Segmental Isolation Based on Stochastic Parametric L-system
}

\author{
Zeinab Abbasia and Ramin Bozorgmehry Boozarjomehry $b$ \\ Department of Chemical and Petroleum Engineering, Sharif University of Technology, P. O. Box \\ 11365-9465, Tehran, Iran \\ a E-mail address: z_abbasi@che.shrif.ir \\ b Corresponding author. Tel.: +98 216616 6445; E-mail address: rbozorgmehry@sharif.edu
}

\section{Abstract}

In the human lung, air is transported from the environment to the respiratory zone by conducting airways containing 60000 airways with different scales. An accurate model of this complicated structure is crucial for studying transport phenomena in the human lung. In the present study, the parametric Lindenmayer System (L-System) has been used to produce the human bronchial tree in the bronchopulmonary segments whose shapes have been obtained based on the medical image processing techniques. The airways propagate into the host segments simultaneously similar to what happens in real life using multi-threads parallelism method. Each module in rewriting rules of the formulated L-System plays the role of an airway with its characteristic properties that have been found almost independently based on an intelligent procedure. This feature enables the method to be less sensitive to change in the host shape and size of the lungs. In addition, the stochastic behavior of the proposed procedure generates slightly different structures with the same statistical properties. Furthermore, the dimensions of the terminal branches are functions of age, which make the proposed method capable of generating the tree structure for the subjects with various ages fulfilling almost all anatomical and physiological requirements. The morphometric characteristics of the generated structure are in good agreement with the corresponding experimental data reported in the literature. The results show that the proposed method outperforms the previously reported models. The accurate three-dimensional model proposed by this study can be used for simulation of fluid distribution, mass and heat transfer, drug delivery, and particle deposition in the human respiratory system.

Keywords: Lung; Bronchial tree; L-System; conducting airways. 


\section{Introduction}

32 There are three and two lobes in the right and left lungs, respectively. Each lobe is also divided

33 into sections called bronchopulmonary segments. Layers of connective tissue separate the

34 segments from each other. Each segment is supplied by a segmental bronchus and bronchial artery

35 and functions in a manner that does not depend on the other segments [1]. The right lung consists

36 of 10 segments whereas the number of left lung segments varies from 8 to 10 . The most common

37 structure of the left lung comprises 8 segments. Air goes into trachea from nose or mouth during

38 breathing. Then it passes through airways into the lungs and backs out again. The airways in the

39 human lungs can be classified into two main parts: conducting airways and respiratory airways.

40 Conducting airways start from the trachea and propagate into the lung as terminal bronchioles.

41 They supply air from the trachea to respiratory units where gas exchange between respiratory

42 airways and blood takes place. The airways have been confined in a limited space resulting in a

43 complicated structure with multi-scales.

44 The conducting airways of the human lungs form a complex asymmetric tree. Mathematical 45 models of the tracheobronchial tree have been widely used to study aerosol deposition[2], gas 46 transport[3-6], ventilation distribution[7, 8], and heat transfer[9]. The accurate modeling of all

47 aforementioned subjects requires appropriate and reliable modeling of the bronchial tree. For this 48 reason, modeling of the bronchial structure has been attracted much attention. Since the problem

49 does not have a unique answer, the previous research tried to present mathematical models with

50 various simplifications in such a way that the statistical properties of the final structure satisfy the

51 anatomical data. Weibel[10] and Yeh and Schum[11] respectively proposed one and five typical

52 symmetric paths' models of the airways obtained based on cast measurement. Although this 
53 approach has the lowest computational cost for studying the aforementioned applications it is not

54 appropriate for studying the effect of anatomical asymmetry.

55 To study the properties of the bronchial tree, the branches should be classified. There are three

56 methods of classification that are commonly used: generation, Horsfield and Strahler order. The

57 first one was used by Weibel[10]. In this approach, counting starts at the trachea, considered as

58 generation 0 or 1 , and increases by one in each bifurcation. Strahler and Horsfield orders [12] are

59 the most common classifying methods for asymmetric trees in which the branches are numbered

60 upward from end branches instead of downward starting with the trachea. Horsfield et.al[13] used

61 parameter delta, the difference between the order of the daughters' branches, to express the

62 asymmetry at dichotomy. This method assumes a uniform degree of asymmetry throughout the

63 tree but the branches with the same order have different diameters or lengths in reality. Koblinger

64 and Hofmann[14] proposed a stochastic asymmetric model for human bronchial tree based on

65 morphometric data of Raabe et.al. [15]. However, all mentioned simplified geometries do not take

66 into account the spatial positions of airways.

67 Kitaoka et.al[16] used nine basic rules and four complementary rules to produce a three-

68 dimensional tree (3-D) representing the bronchial tree of the lungs. They used a power-law

69 relationship between the flow dividing ratio and diameter. To find the branching angle, the relation

70 proposed by Murray[17] was employed, which has been derived based on the minimization of

71 power dissipation and is a function of the flow dividing ratio. They assigned a value to the branch

72 length that was three times its diameter. Their deterministic model was very sensitive to the initial

73 value of model parameters and unable to generate various structures with identical structural

74 properties. Tahwai et.al [18] proposed a model for the construction of a 3-D bronchial tree in each

75 lobe based on Monte Carlo method. They distributed seed points on the host volume and space 
was divided into two sub-volumes by a dividing plane. The branching angle was found based on a

77 growing daughter branch toward the center of mass of the seed points in each subvolume. They

78 assumed that the length of each branch to be a predefined fraction of the distance between the

79 starting point of the branch and the center of the mass. The diameters were randomly assigned to

80 all branches after the whole structure was constructed based on the Horsfield order with a

81 coefficient of variation (defined as the ratio of standard deviation to the mean) of 0.1 . Tahwai et.al

82 [19] obtained the upper parts of the bronchial tree using Multi-Detector X-ray-row CT (MDCT)

83 and used the model of [18] to complete the structure. They also improved the previous model by

84 a change in the number of seed points, regrouping them throughout the generation process, and

85 using Strahler order for the determination of branches diameters. Bordas et.al [20] used the same

86 approach for the generation of the bronchial tree for healthy and asthmatic patients. For assignment

87 of diameter to the branches, they used the terminal segmented branches as base branches instead

88 of the trachea. The incorporation of features of diseases was not observed in their model

89 parameters, and the difference between the structure of healthy subjects and patients only arises

90 from the dissimilarity in the upper airways obtained by MDCT. Montesantos et.al [21] used

91 Tawhai et.al[19] model along with HRCT data for producing deterministic three-dimensional

92 branches into lobes. They assigned diameter to the branches by using a predefined value for length-

93 to-diameter ratio instead of using Horsfield or Strahler order. The deterministic behavior, assigning

94 diameter after construction, needing detailed knowledge before generating the structure, and

95 assumption of one dimensional (1-D) branches during generation process are the main

96 shortcomings of such models. Davoodi and Bozorgmehry[22] used a stochastic rule-based method

97 to generate the structure of human conducting airways using Lindenmayer-system (L-system).

98 They used a similar approach of Kitaoka et.al[16] for finding diameter and angle. Although the

99 length was found independently in this method, the branching angle and diameter are correlated 
causing the model to be sensitive to the model parameters and change in the host geometry. For

101 instance, if their model with the proposed parameters is used to obtain the conducting airways in

102 the bronchopulmonary segments, the structure will have too small terminal bronchioles. Another

103 shortcoming of their work is that they do not take into account the space occupied by other

104 branches in the procedure of finding the length of the growing branch.

105 The shape of the host volume significantly affects the structure of the generated tree. In this study,

106 we try to produce the tracheobronchial tree in the bronchopulmonary segments in contrast to the

107 previous models that generate the tree in the lungs or lobes. In the present work, we use parametric

108 L-System to generate the human bronchial tree because based on this approach it is possible to

109 define a branch as a function of its characteristic properties, i.e. diameter, length, and branch

110 orientation, and consider the growth of the tree over time. The major difference of the proposed

111 model with the previous ones is that the characteristic properties of the branches are found

112 independent of each other based on an intelligent procedure in such a way that the anatomical

113 constraints are satisfied. This approach makes the model so flexible to generate the bronchial tree

114 more realistically in quite various host volumes with the same rules and model parameters. In

115 addition, this approach makes model capable of producing the structure for other species with a

116 few prior information in contrast to that proposed by Tawhai et.al[19] that needs the diameter

117 reduction as a function of Strahler order. Another unique advantage of the proposed method is that

118 the branches do not grow in the bronchopulmonary segments independently and the branches of

119 all segments propagate in parallel which is what happens in reality. In other words, the positions

120 of branches near the segments' interfaces affect the orientation of growing branches. Furthermore,

121 the method is developed so that the airways grow 3-D quite similar to what happens in reality

122 while this is not the case the previous works in which the airways grow 1-D. 
123 The remaining sections of this paper are organized as follows. Section 2 presents the

124 methodology of finding bronchopulmonary segments and the mathematical formulation of the

125 growing structure in the host volumes. In Section 3, the production rules and the proposed

126 procedure for finding parameters of parametric L-system are discussed. In Section 4, the

127 performance of the proposed model is evaluated by comparison of the model results with those

128 reported in the previous studies. Finally, discussion and concluding remarks are given in Sections.

1295 and 6 , respectively.

\section{Problem Statement}

131 To construct the bronchopulmonary tree, the boundaries of each bronchopulmonary segment

132 should be first determined. The next section goes through the procedure by which the

133 bronchopulmonary segments are built. Sections. 2.2 and 2.3 elaborate the general mathematical

134 formulation of the problem and growth based formulation of the problem using L-System.

136 Bronchopulmonary segments separate from each other by thin connective tissue, which is not

137 visible in CT images. On the other hand, since the boundaries between lobes are visible in CT

138 images; the lobes are segmented out using chest imaging platform module of 3DSlicer which is a

139 free software for image analysis. After this lobe segmentation, bronchopulmonary segments

140 fissures were segmented manually using their anatomical positions, lobe boundaries and

141 considering the fact that each segment is fed by only one airway and artery. The process of 142 segmentation of bronchopulmonary segments using predefined fissures was done by the 143 segmentation module of 3DSlicer software. At the end of these steps, the surface of each 144 bronchopulmonary segment is obtained as a set of triangular grids. It should be noted that the 
145 boundaries of the bronchopulmonary segments are only manually found once. For other subjects,

146 they can be estimated using the lobes' boundaries assuming that the relative position of the

147 segments' boundaries in the corresponding lobes remain unchanged.

\subsection{Mathematical formulation}

149 Since the problem belongs to the category of inverse problems and there is no one to one 150 mapping between the model parameters and the observations, the problem has no unique solution.

151 In what follows, we present various models and show that the model with the least number of 152 parameters is suitable because there is not enough data to determine many parameters.

153 The human bronchial tree can be described by a simple directed graph 1 whose edges are three-

154 dimensional. All nodes and edges of the graph should be determined in such a way that the 155 structure satisfies all anatomical and physiological constraints of a living organ. Therefore, the 156 problem can be defined by:

$$
\begin{aligned}
& \text { minimize } f(G(N, E)): N \times E \rightarrow \mathbb{R}^{+} \\
& \text {subject to: } \tilde{C}(G(N, E))<\tilde{\varepsilon}
\end{aligned}
$$

157 where $G(N, E)$ is a simple directed graph with a set of nodes $N$ and a set of three dimensional

158 directed edge E. $f$ and $\tilde{C}$ are the norms of the vector of objective functions $\tilde{F}$, and vector of 159 constraints whose elements are members of $\mathbb{R}^{+}$.

\subsection{Growth based formulation}

161 There are about 60000 conducting airways in the human bronchial tree[23] and thus the number

162 of decision variables of the problem is 240000 calculated by considering four decision variables

\footnotetext{
1 In mathematics, simple directed graph is a directed graph without loop.
} 
163 (i.e. the diameter and the endpoint position) for each branch. It is difficult to find such a large

164 number of variables in such a way that the proposed algorithm remains robust in the presence of 165 small disturbances or deformations. Therefore, it is essential to reduce the number of decision 166 variables in such a way that the model remains accurate and predictable. For this purpose, the 167 graph $\mathrm{G}$ can be obtained using a rule-based method such as Lindenmayer system. This method and 168 its application in the modeling of the structure are described in the rest of this section.

169 Lindenmayer system or L-system was introduced and developed by Aristid Lindenmayer[24, $17025]$. It is a type of formal grammar and a parallel rewriting system that uses production rules or a 171 set of grammars to produce branching structures. L-systems have been used to describe the growth 172 process of plants[26, 27], model morphology of organisms[22, 28], and the production of self173 similar fractals. It can be defined as a triplet $\mathrm{L}(V, \omega, P)$. L-System consists of four main parts:

$174 \quad-\quad$ : A set of symbols that are used to make string. It consists of both variables, elements that 175 can be replaced, and terminal or constants, the elements that cannot be replaced. The set of 176 all words and set of all nonempty words are denoted by $V^{*}$ and $V^{+}$, respectively.

$177-\omega \in V^{+}:$An axiom that is an initial string from which the branching starts.

$178-P \subset V \times V^{*}:$ A set of production rules that are employed to make strings from each 179 symbol. It consists of a predecessor and a successor and is written in the following format.

$$
p: \text { predecessor } \rightarrow \text { successor }
$$

180 The symbol " $\rightarrow$ " is used to separate the predecessor and successor.

181 - Turtle interpretation: a mechanism that interprets the strings into geometric shapes. 
182 Lindenmayer used brackets "[" and "]" to produce axial trees. These brackets are employed to 183 enable L-system to produce branching structure. The brackets "[" and "]" are not included in the 184 set $\mathrm{V}$, therefore the set $\mathrm{V}$ is extended to set $V_{B}=V \bigcup\{[]$,$\} for bracketed L-system.$

185 If the graph representing the bronchial tree is obtained based on L-system, the formulation of the 186 problem will be:

$$
\begin{array}{ll}
\text { minimize } & f(\mathrm{~L}(V, \omega, P)) \\
\text { subject to: } & \tilde{C}(\mathrm{~L}(V, \omega, P))<\tilde{\varepsilon}
\end{array}
$$

187 To generate the structure of the human bronchial tree using the above approach, the number of 188 production rules, i.e. decision variables, is numerous because the bronchial tree has a non-uniform 189 structure. Therefore, it is of interest to reduce the number of production rules. For this purpose, the 190 parametric L-system is used to generate the tree with a minimum number of decision variables that 191 include both production rules and parameters.

192 In parametric L-system predecessors and successors are parametric strings with letters from set $193 \mathrm{~V}$ and formal parameters, real-valued parameters appearing in words, from set $\Sigma$. Any module $194 F\left(a_{1}, a_{2}, \ldots, a_{n}\right)$ with letter $F \in V$ and parameters $a_{1}, a_{2}, \ldots, a_{n} \in \mathbb{R}$ belongs to set $V \times \mathbb{R}^{*}$. 195 Parametric L-system is denoted by quadruplet $\mathrm{L}(V, \Sigma, \omega, P)$ where:

$196 \omega \in\left(V \times \mathbb{R}^{*}\right)^{+}$is axiom.

$197 P \subset\left(V \times \Sigma^{*}\right) \times \mathbb{C}(\Sigma) \times(V \times \mathcal{E}(\Sigma))^{*}$ is a set of production rules.

198 where $\left(V \times \mathbb{R}^{*}\right)^{+}$and $\mathbb{C}(\Sigma)$ are a set of nonempty modules and a set of logical expressions, 199 respectively. $\mathcal{E}(\Sigma)$ denotes the set of all procedures for finding parameters. Symbol "*” above any 200 set B denotes the set of all finite combinations of members of $\mathrm{B}$. The parametric production rule 201 is written in the following format. 
p: predecessor(parameters): condition $\rightarrow$ successor(parameters)

202 Where the symbol ":" is used to separate the condition and predecessor. Any formal parameters

203 may appear in the conditions. The reader is referred to the reference [24] for further details on

204 different types of L-System. Using parametric L-system, Eq. (2.3) can be reduced to:

minimize $f(\mathrm{~L}(V, \Sigma, \omega, P))$

subject to: $\tilde{C}(\mathrm{~L}(V, \Sigma, \omega, P))<\tilde{\varepsilon}$

205 All structures produced by the same deterministic L-system are identical. To generate the bronchial

206 structure for various people, it is necessary to randomize parameters so that it considers case-to-

207 case variations and preserves statistical properties of the structure. In the next section, the process

208 of generating tracheobronchial structure using parametric L-system with random parameters is 209 presented.

\section{Application of Parametric L-System in the Modelling od Conducting Airways}

211 Trachea branches into main bronchi which are then branching into lobar (secondary) bronchi.

212 Lobar bronchi further divide into segmental (tertiary) bronchi which branch into smaller airways

213 called bronchiole connected to terminal bronchiole feeding respiratory zone. Conducting airways

214 can be divided into two main sections. The first section extends from the trachea to segmental

215 bronchi. The second section comprises segmental bronchi to terminal bronchioles. All branches

216 with identical segmental bronchus are generated into their corresponding bronchopulmonary

217 segment and the generation of branches in each segment occurs in parallel. Parametric L-system

218 is used to produce the branches of each mentioned section. Each airway can be determined using

219 its parent, diameter, length, and orientation. Parameters of parametric L-system should be

220 determined by an intelligent procedure to satisfy anatomic constraints for all cases. The decision

221 variables of the proposed procedure should be determined in such a way that the objective function 
222 of Eq. (2.5) is minimized. Each member of the vector $\tilde{F}$ corresponds to the difference between the

223 statistical property of simulated structure and its reference value according to the following Eq.

224 (3.1).

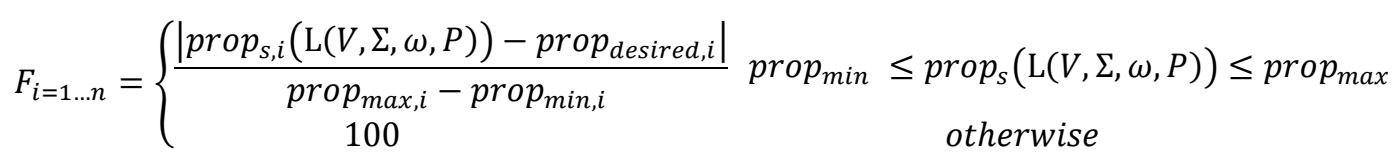

225 Where, $\operatorname{prop}_{s, i}$ is a function that calculates the corresponding property of the simulated structure.

226 The list of geometrical properties considered in Eq. (3.1) is given in Table 1. The other properties

227 such as $d_{\text {min }} / d_{p}$ (dimeter of minor daughter to diameter of parent $), d_{\text {min }} / d_{\text {maj }}$ (dimeter of minor daughter

228 to diameter of major daughter), HRb (Horsfield branching ratio), HRd (Horsfield diameter ratio),

$229 \mathrm{HRl}$ (Horsfield length ratio), $d_{T B}$ (diameter TB) and $L_{T B}($ length of TB) are used for validation of

230 the obtained structure. The evaluation results are presented in Section 4. In addition, the proposed

231 method fulfills the following requirements:

232 1. Each branch is three dimensional during the growth process.

233 2. Every parent branch is divided into two daughter branches.

234 3. The diameters of the daughter branches are less than that of their parent.

235 4. The branches intersect neither the host boundary nor each other.

236 5. The minimum angle between daughter branches is $10^{\circ}$.

237 Table 1. List of geometrical properties and their corresponding acceptable range considered in the objective function

\begin{tabular}{|c|c|c|c|c|c|c|}
\hline prop $_{i}$ & definition & Reported values & reference & $\operatorname{prop}_{\min , i}$ & prop $_{\max , i}$ & prop $_{\text {desired }}$ \\
\hline$l / d$ & length to diameter ratio & $3.25-3.75$ & {$[29,30]$} & 2.9 & 4.1 & 3.5 \\
\hline$d / d_{p}$ & Dimeter to diameter of parent & $0.83-0.88$ & {$[29,31,32]$} & 0.75 & 0.92 & 0.85 \\
\hline$d_{m a j} / d_{p}$ & $\begin{array}{l}\text { Dimeter of major daughter to } \\
\text { diameter of parent }\end{array}$ & 0.86 & [33] & 0.81 & 0.95 & 0.89 \\
\hline$l / l_{p}$ & length to length of parent & 0.85 & {$[29,32]$} & 0.76 & 0.94 & 0.9 \\
\hline$\theta$ & Branching angle & $25-47$ & {$[31,34]$} & 35 & 50 & 43 \\
\hline $\mathrm{SRb}$ & Strahler branching ratio & $2.51-2.81$ & [35] & 2.45 & 2.95 & 2.8 \\
\hline SRd & Strahler diameter ratio & $1.35-1.5$ & {$[35,36]$} & 1.3 & 1.6 & 1.41 \\
\hline SRl & Strahler length ratio & $1.33-1.55$ & {$[35,36]$} & 1.3 & 1.6 & 1.41 \\
\hline
\end{tabular}




\subsection{Central airways}

239 As mentioned before, the number of bronchopulmonary segments varies from 18 to 20 . This

240 causes the structure of central airways to vary among various people. To find the most common

241 structure, $50 \mathrm{CT}$ images of various people were studied[37, 38]. We found that the most common

242 structure is a lung with 18 segmental bronchi as shown in Fig. 1. Table 2 shows the name of each

243 segment fed by segmental bronchus indicated in Fig. 1.

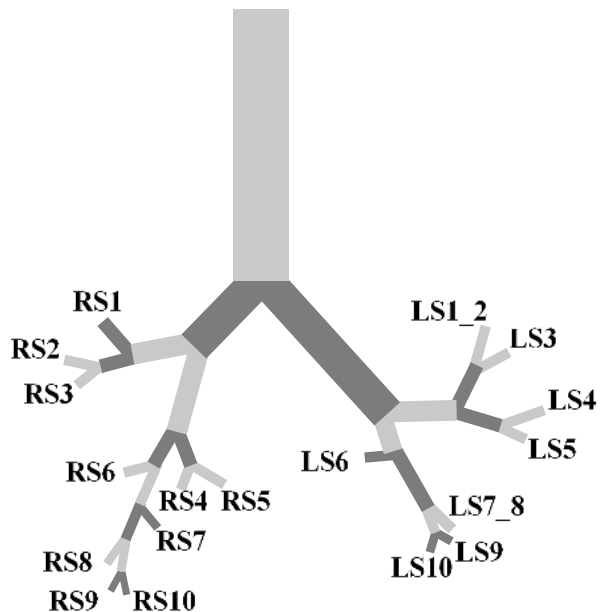

Fig. 1 Central airways

Table 2. bronchopulmonary segments and corresponding segmental bronchus

\begin{tabular}{|c|c|c|c|c|c|}
\hline \multicolumn{3}{|c|}{ Right Lung } & \multicolumn{3}{|c|}{ Left Lung } \\
\hline Branch number & Lobe & Segment & Branch number & Lobe & Segment \\
\hline RS1 & \multirow{3}{*}{ Upper Lobe } & Apical & LS1 2 & \multirow{5}{*}{ Upper Lobe } & Apicoposterior \\
\hline RS2 & & Posterior & & & \\
\hline RS3 & & Anterior & LS3 & & Anterior \\
\hline RS4 & \multirow{2}{*}{ Middle Lobe } & Medial & LS4 & & Superior lingular \\
\hline RS5 & & Lateral & LS5 & & Inferior lingular \\
\hline RS6 & \multirow{5}{*}{ Lower Lobe } & Superior & LS6 & \multirow{5}{*}{ Lower Lobe } & Superior \\
\hline RS7 & & Medial & LS7 & & Anteromedial \\
\hline RS8 & & Anterior & & & \\
\hline RS9 & & Lateral & LS8 & & Lateral \\
\hline RS10 & & Posterior & LS9 & & Posterior \\
\hline
\end{tabular}


250 rules. Because the central structure is slightly different from the one reported by Davoodi and

251 Boozarjomehry [22], their production rules are modified to produce the central structure shown in

252 Fig. 1. The modified production rules are shown in Eq. (3.2).

$$
\begin{aligned}
& \omega: T\left(l_{0}, d_{0}\right) \\
& p_{1}: T\left(l_{p}, d_{p}\right) \rightarrow+(\delta) \backslash(\delta) E\left(\alpha l_{p}, \beta d_{p}\right)\left[+(\delta) \backslash(\delta) B\left(\alpha L, \beta d_{p}\right)+(\delta) \backslash\right. \\
& \left.(\delta) C\left(\alpha l_{p}, \beta d_{p}\right)\right]\left[+(\delta) \backslash(\delta) G\left(\alpha l_{p}, \beta d_{p}\right)+(\delta) \backslash(\delta) F\left(\alpha l_{p}, \beta d_{p}\right)\right] \\
& p_{2}: B\left(l_{p}, d_{p}\right) \rightarrow E\left(\alpha l_{p}, \beta d_{p}\right)\left[+(\delta) \backslash(\delta) K\left(\alpha l_{p}, \beta d_{p}\right)++(\delta) \backslash(\delta) M\left(\alpha l_{p}, \beta d_{p}\right)\right] \\
& p_{3}: C\left(l_{p}, d_{p}\right) \rightarrow E\left(\alpha l_{p}, \beta d_{p}\right)\left[+(\delta) \backslash(\delta) M\left(\alpha l_{p}, \beta d_{p}\right)\right]\left[+(\delta) \backslash(\delta) K\left(\alpha l_{p}, \beta l_{p}\right)[+(\delta) \backslash\right. \\
& (\delta) K\left(\alpha l_{p}, \beta d_{p}\right)\left[+(\delta) \backslash(\delta) K\left(\alpha l_{p}, \beta d_{p}\right)+(\delta) \backslash(\delta) M\left(\alpha l_{p}, \beta d_{p}\right)\right] \\
& p_{4}: G\left(l_{p}, d_{p}\right) \rightarrow E\left(\alpha l_{p}, \beta d_{p}\right)\left[+(\delta) \backslash(\delta) K\left(\alpha l_{p}, \beta d_{p}\right)\left[+(\delta) \backslash(\delta) K\left(\alpha l_{p}, \beta d_{p}\right)+\right.\right. \\
& \left.(\delta) \backslash(\delta) M\left(\alpha l_{p}, \beta d_{p}\right)\right] \\
& p_{5}: F\left(l_{p}, d_{p}\right) \rightarrow E\left(\alpha l_{p}, \beta d_{p}\right)\left[+(\delta) \backslash(\delta) M\left(\alpha l_{p}, \beta d_{p}\right)\right]\left[+(\delta) \backslash(\delta) M\left(\alpha l_{p}, \beta d_{p}\right)\right] \\
& p_{6}: M\left(l_{p}, d_{p}\right) \rightarrow E\left(\alpha l_{p}, \beta d_{p}\right)\left[+(\delta) \backslash(\delta) A\left(\alpha l_{p}, \beta d_{p}\right)\right]\left[+(\delta) \backslash(\delta) A\left(\alpha l_{p}, \beta d_{p}\right)\right] \\
& p_{7}: K\left(l_{p}, d_{p}\right) \rightarrow E\left(\alpha l_{p}, \beta d_{p}\right)\left[+(\delta) \backslash(\delta) A\left(\alpha l_{p}, \beta d_{p}\right)\right]
\end{aligned}
$$

253 where $l_{p}, d_{p}, \delta, \beta$, and $\alpha$ are branch length, diameter, orientation angle and ratio of diameter and 254 length of daughter to its parent, respectively. $+(\delta)$ and $\backslash(\delta)$ denotes orientation in azimuthal and 255 polar direction. If $\delta$ is positive (negative) the rotation will be counterclockwise (clockwise). $256 \mathrm{~T}\left(l_{p}, d_{p}\right)$ indicates a cylindrical airway with the length of $l_{p}$ and the diameter of $d_{p} . T\left(l_{0}, d_{0}\right)$ 257 corresponds to the trachea. Actions of characters E, B, C, G, F, K, M, and N are similar to T. 258 Character "A" represents an inactive branch, at which the growth is stopped. The current position 259 and orientation are saved when turtle interpretation encounters the "[" 260 turtle interpretation encounters "]". Rewriting the rules should continue until no active branch 261 remains. Central airways are produced by three generations of growth based on the production 262 rules. 
All parameters are obtained by the analysis of CT images taken from Exact09 website [37].

264 This dataset contains 40 CT scan images. The datasets used in this study are case01 and case 37

265 which belong to adult male subjects. The airway segmentation module of 3DSlicer software was

266 used to extract central airways from CT images. The distance between two bifurcation points was

267 considered for branch length and average diameter of branch used as diameter. Polar and azimuthal

268 angles were estimated using the given branch orientation. All segmental bronchi (last branches of

269 central airways) are used as the axiom of the second section.

\subsection{Dichotomous structure}

To generate the dichotomous tree, parametric L-System is used. Because the rules proposed by

272 Davoodi and Boozarjomehry [22]are based on the bifurcating concept, we used their rules given

273 in Eq. (3.1). In each generation, a parent airway divides into two daughter airways in a single

274 branching plane. It should be noted that these rules are modified to include any rotation angle,

275 which is the angle between two successive branching planes. In addition, the process of finding

276 the parameters is different.

$$
\begin{aligned}
& \omega: F\left(l_{0}, d_{0}, N_{0}, 0\right) \\
& P_{1}: F\left(l_{p}, d_{p}, N_{p}, 0\right) \rightarrow E\left(l_{p}, d_{p}\right)\left[-\left(\theta_{1}\right) F\left(\Gamma\left(D, \gamma_{1} d_{p}\right), \gamma_{1} d_{p}, N(\varphi), c\right)\right]\left[+\left(\theta_{2}\right) F\left(\Gamma\left(D, \gamma_{2} d_{p}\right), \gamma_{2} d_{p}, N(\varphi), c\right)\right] \\
& P_{2}: F\left(l_{p}, d_{p}, N_{p}, 1\right) \rightarrow E\left(l_{p}, d_{p}\right)\left[-\left(\theta_{1}\right) A\left(\Gamma\left(D, \gamma_{1} d_{p}\right), \gamma_{1} d_{p}, N(\varphi)\right)\right]\left[+\left(\theta_{2}\right) F\left(\Gamma\left(D, \gamma_{2} d_{p}\right), \gamma_{2} d_{p}, N(\varphi), c\right)\right] \\
& P_{3}: F\left(l_{p}, d_{p}, N_{p}, 2\right) \rightarrow E\left(l_{p}, d_{p}\right)\left[-\left(\theta_{1}\right) F\left(\Gamma\left(D, \gamma_{1} d_{p}\right), \gamma_{1} d_{p}, N(\varphi), c\right)\right]\left[+\left(\theta_{2}\right) A\left(\Gamma\left(D, \gamma_{2} d_{p}\right), \gamma_{2} d_{p}, N(\varphi)\right)\right] \\
& P_{4}: F\left(l_{p}, d_{p}, N_{p}, 3\right) \rightarrow E\left(l_{p}, d_{p}\right)\left[-\left(\theta_{1}\right) A\left(\Gamma\left(D, \gamma_{1} d_{p}\right), \gamma_{1} d_{p}, N(\varphi)\right)\right]\left[+\left(\theta_{2}\right) A\left(\Gamma\left(D, \gamma_{2} d_{p}\right), \gamma_{2} d_{p}, N(\varphi)\right)\right]
\end{aligned}
$$

$277 \theta$ and $\varphi$ are branching angle, the angle between the branch and its parent, and rotation angle, 278 respectively. $N_{p}$ and $N$ are the normal vectors of the parents' plane and daughters' plane. The 279 schematic of two successive branching planes is shown in Fig. 2. $l_{p}$ and $d_{p}$ are the length and 280 diameter of the parent branch. $\gamma$ is the ratio of branch diameter to that of its parent. 
281 Turtle interpretation of the above rules is described in Table 3. $\Gamma(D, d)$ is a function for finding

282 branch length based on parameter $\mathrm{D}$ and the branch diameter $d$. Branching procedure terminates

283 at A which is terminal bronchiole (TB). The value of parameter c determines whether a branch

284 terminates or not. The procedure of finding parameter c depends on the extent of our knowledge

285 of the system, i.e. the tracheobronchial tree of humans or other species. If enough data are

286 available about the length and diameter of terminal bronchioles, their values are used as

287 termination criteria; otherwise, a reasonable distance to the corresponding boundary is considered

288 for this purpose. Based on previous measurements on the human bronchial tree, the diameter and

289 length of TB vary from $0.432 \pm 0.035 \mathrm{~mm}[39]$ to $0.66 \pm 0.04 \mathrm{~mm}[40]$, and from $0.8 \pm 0.35 \mathrm{~mm}$ [23]

290 to $1.7 \mathrm{~mm}[31]$, respectively. A branch is considered TB if its diameter and length are both less

291 than $d_{T B, \max }$ and $L_{T B, \max }$, respectively. Since maximum measured values for the parameters have

292 not been reported in the previous studies, the values of $d_{T B, \max }$ and $L_{T B, \max }$ should be higher than

$2930.66+0.04$ and 1.7, respectively. To prevent the production of very small TBs, these threshold

294 values have been obtained through ad-hoc iterative method. The appropriate values of $d_{T B, \max }$ and

$295 L_{T B, \max }$ are $0.8 \mathrm{~mm}$ and $1.8 \mathrm{~mm}$, respectively.

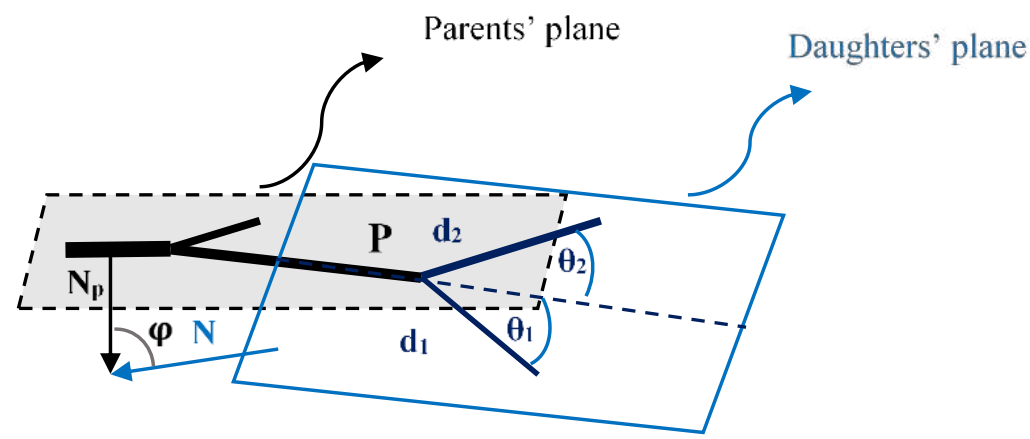

296

Fig. 2 Schematic of two successive branching planes

Table 3 symbols of commands in turtle interpretation for dichotomous rewriting rules

Command

Action 
$F(l, d)_{l} \quad$ move forward and draw a cylinder with length of $l$ and diameter of $d$

$\mathrm{N}(\varphi) \quad$ Calculate the normal vector of daughters' plane and turn parent plane by angle $\varphi$

$-(\theta) \quad$ Turn by angle $\theta$ in the clockwise direction

$+(\theta) \quad$ Turn by angle $\theta$ in the counter-clockwise direction

$C \quad$ Its value determines if the branching process continues from the daughter(s) branch or not

1 action of characters $\{\mathrm{A}, \mathrm{E}\}$ is similar to $\mathrm{F}$ but the branching process terminates in $\mathrm{A}$.

300 In the human bronchial tree, each branch divides into two branches with a reduction in diameter.

301 Mauroy et.al [32] showed that the best structure minimizing total viscous dissipation is a fractal

302 whose dimension is 3 . In this ideal tree size ratio, the ratio of the daughters' dimensions to the

303 corresponding dimensions of their parent, is $(1 / 2)_{1 / 3} \sim 0.79$. In addition, they demonstrated that a

304 physically optimum tree does not have physiological robustness of the human bronchial tree. The

305 human bronchial tree has an average size ratio of 0.85 , which is larger than the physically optimum

306 value, and with such a size ratio, the volume of the human bronchial tree is too large and its

307 resistance is too small. Some previous research aimed to design a bronchial tree based on

308 minimizing the volume and total resistance[41] or bifurcating resistance [16, 22]. However,

309 physical optimization of the bronchial tree may be dangerous [32]. On the other hand, it is

310 necessary to model the bronchial tree to satisfy the physiological constraints. In what follows, we

311 suggest an intelligent procedure for finding the parameters of L-System to fulfill the physiological

312 and anatomical requirements of dimensions and orientations.

313 Parameters $\gamma_{1}$ and $\gamma_{2}$ must be less than 1 since the daughters' diameters are always smaller than

314 their parents. Murray[17] showed that $\mathrm{Q}=\mathrm{Cd}_{3}$ minimizes the power dissipation in an ideal tree

315 assuming laminar flow. Uylings [42] extended the equation to $\mathrm{Q}=\mathrm{Cd}$ where ' $\mathrm{n}$ ' is diameter

316 exponent and depends on flow regime, $n=3$ for laminar and $n=2.34$ for the turbulent regime.

317 Assuming a flow ratio ' $r$ ' the parameters $\gamma_{1}$ and $\gamma_{2}$ are: 


$$
\gamma_{1}=r^{\frac{1}{n}} \quad \gamma_{2}=(1-r)^{\frac{1}{n}}
$$

318 Kitaoka et.al [16] found that $n=2.8$ leading to the best structure. Flow is not turbulent in all parts

319 of the dichotomous structure, so the value should be greater than 2.33. On the other hand, the 320 viscous forces are only dominant in the periphery of conducting airways thus the value should be 321 less than 3. ' $r$ ' is flow dividing ratio defined as the ratio of smaller flow in the daughters to that in 322 the parent. In this study, it varies from 0.15 to 0.5 indicating the structural asymmetry. As this 323 parameter gets closer to 0.5 the structure gets more symmetric. Since the human lung structure 324 becomes more symmetric with the generation, the parameter approaches to 0.5 with increasing 325 generation. Hence, this parameter is calculated by a normal distribution at each generation in such 326 a way that it remains between $r_{\min }$ and $r_{\max }$, where $\mathrm{r}_{\min }$ and $\mathrm{r}_{\max }$ are functions of generation and 327 change after some generations to propel the structure toward symmetrizing.

328 To find the branch length an initial length $(\mathrm{Lm}>\mathrm{d})$ is considered. It is then examined whether 329 this branch intersects the Segment boundary and other branches during its growth or not. In 330 addition, this branch should be sufficiently far away from the Segment boundary so that there is 331 enough space for the growth of other conducting airways and the acinar region. Therefore, the 332 parameter $\mathrm{D}$ is defined as the minimum of the distance between the branch and other branches and 333 a fraction $\left(\mathrm{r}_{\mathrm{d}}\right)$ of its distance to the boundary. The latter was used to ensure the availability of 334 enough space for growth of acinar region which was ignored in the model of Davoodi and 335 Boozarjomehry[22] . Afterward, the branch length is adjusted in a value less than the minimum 336 of D and Lm according to Eq. (3.5). Kiatoka[16] suggested that the length to distance ratio ranges 337 from 0.17 to 0.34 by examining many computed tomography images. Because the length is 338 reduced using Eq. (3.5), the value of $r_{d}$ is set to 0.5 .

$$
\Gamma(D, d)=\min (\operatorname{Lm}, D) \min (\exp (-\omega * D / d), 0.85)
$$


where $\omega$ and is a measure of length reduction and is set at 0.04. Davoodi and Boozarjomehry[22]

340 used a similar equation for length reduction. They decreased the length by a factor of $\exp (-\omega / \mathrm{D})$,

341 where D was a minimum distance of the new branch to other branches and the boundary. However,

342 this equation couldn't be used for different sizes of lungs originated from various ages because the

343 parameter $\omega$ was not dimensionless. Therefore, the expression is age-dependent and results in

344 more reduction in length for shorter branches. Hence, in their model, some parameters should be

345 changed to make the model capable of the tracheobronchial tree construction for subjects with

346 various ages successfully.

347 A mathematical model is required for the boundaries of all bronchopulmonary segments to 348 calculate the distance of each branch to the corresponding boundary. Davoodi and 349 Boozarjomehry[22] used the thin-plate smoothing spline method to describe the boundaries and 350 subsequently calculated the distance between each branch and lobe boundaries. However, the 351 method cannot accurately describe the non-convex characteristic of bronchopulmonary segments' 352 boundaries. Therefore, each boundary is discretized by the triangular elements. The distance to the 353 boundary is the minimum of the distance of the branch to all triangles. To find the distance, it is 354 necessary to obtain the intersection of the branch with all triangular cells; accordingly, its 355 calculation algorithm should be fast enough to avoid the computational time and demand. For this 356 purpose, the ray-triangle intersection algorithm proposed by Moller and Trumbor [43] and Havel 357 and Harout [44] were examined, which are the fastest algorithms. Finally, the algorithm suggested 358 by Moller and Trumbor has been used. To further decrease the calculation time corresponding to 359 the evaluation of branch distance to the boundary, the surface mesh became coarse as much as 360 possible. For this purpose, the triangular cells of different sizes have been used to keep the shape 361 of the segments unchanged as, for instance, indicated in Fig. 3. It should be noted that this 
362 algorithm has been also used to obtain the distance between each branch with its surrounding

363 branches. For this purpose, each branch is surrounded by a cuboid which can be described by 12

364 triangular cells. Therefore, the distance can be approximated by the minimum distance of the

365 growing branch to the triangular cells of other branches. In addition, before the calculation of the

366 intersection of the branch with the other ones, the probability of its collision with them is examined.

367 In other words, for cases where the collision probability is not zero, the ray-triangle intersection

368 algorithm has been used. The intersection probability will be zero if the distance between the

369 centers of the branch and another branch is less than half of the sum of the length and diameter of

370 both branches. This makes the algorithm 20 times faster.

371

372

373

374

375

376

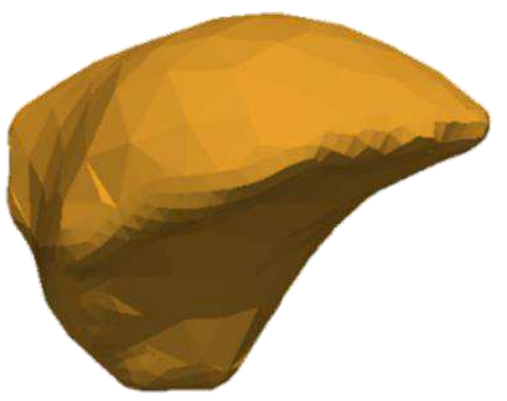

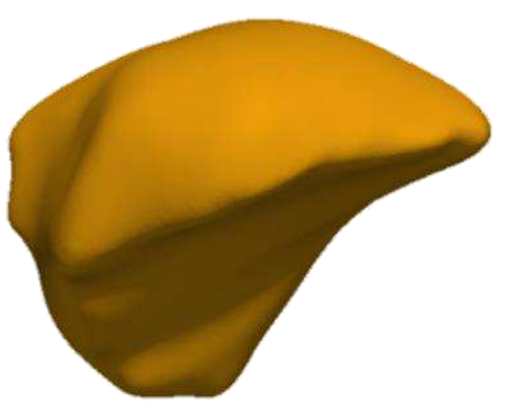

Fig. 3 The structure obtained based on a coarse mesh (left) and extra fine (right) one for the apical segment of the right upper lobe

377 Fig. 2. Accordingly, a normal vector to daughters' plane must satisfy the following equations.

$$
\begin{aligned}
& N \cdot P=0 \\
& N \cdot N_{p}=|N|\left|N_{p}\right| \cos (\varphi) \\
& |N|=1
\end{aligned}
$$


where $\mathrm{P}, \mathrm{N}$, and $\mathrm{N}_{\mathrm{p}}$ are branch orientation of parent, normal vector to daughters' plane, and a normal vector to the parents' plane, respectively. By solving the above equations, the elements of

$380 \quad \mathrm{~N}$ are obtained by:

$$
\begin{aligned}
& A=\left(\left|N_{p} \times P\right|^{2}-\left(|P|\left|N_{p}\right| \cos (\varphi)\right)^{2}\right)^{0.5} N \cdot N_{p}=|N|\left|N_{p}\right| \cos (\varphi) \\
& N_{x}=-A\left|N_{p} \times P\right|_{x}+\left(N_{p x}\left(P_{y}^{2}+P_{z}^{2}\right)-N_{p y} P_{x} P_{y}-N_{p z} P_{x} P_{z}\right)\left|N_{p}\right| \cos (\varphi) \\
& N_{y}=-A\left|N_{p} \times P\right|_{y}+\left(N_{p y}\left(P_{x}^{2}+P_{y}^{2}\right)-N_{p x} P_{x} P_{y}-N_{p z} P_{y} P_{z}\right)\left|N_{p}\right| \cos (\varphi) \\
& N_{z}=-A\left|N_{p} \times P\right|_{z}+\left(N_{p z}\left(P_{x}^{2}+P_{y}^{2}\right)-N_{p x} P_{x} P_{z}-N_{p y} P_{y} P_{z}\right)\left|N_{p}\right| \cos (\varphi)
\end{aligned}
$$

381 Now, branch orientation can be determined by solving Eq. (3.8) and there are two possible 382 solutions which are given in Eqs. (3.9-3.10) for both clockwise and counter-clockwise directions, 383 respectively.

$$
\begin{aligned}
& N \cdot d=0 \\
& P \cdot d=|d||P| \cos (\theta) \\
& |d|=1
\end{aligned}
$$

384

$$
\begin{aligned}
& A=\left(|N \times P|^{2}-(|P||N| \cos (\theta))^{2}\right)^{0.5} \\
& d_{x}=A|N \times P|_{x}+\left(P_{x}\left(N_{y}^{2}+N_{z}^{2}\right)-P_{y} N_{x} N_{y}-P_{z} N_{x} N_{z}\right)|P| \cos (\theta) \\
& d_{y}=A|N \times P|_{y}+\left(P_{y}\left(N_{x}^{2}+N_{y}^{2}\right)-P_{x} N_{x} N_{y}-P_{z} N_{y} N_{z}\right)|P| \cos (\theta) \\
& d_{z}=A|N \times P|_{z}+\left(P_{z}\left(N_{x}^{2}+N_{y}^{2}\right)-P_{x} N_{x} N_{z}-P_{y} N_{y} N_{z}\right)|P| \cos (\theta)
\end{aligned}
$$

385

$$
\begin{aligned}
& A=\left(|N \times P|^{2}-(|P||N| \cos (\theta))^{2}\right)^{0.5} \\
& d_{x}=-A|N \times P|_{x}+\left(P_{x}\left(N_{y}^{2}+N_{z}^{2}\right)-P_{y} N_{x} N_{y}-P_{z} N_{x} N_{z}\right)|P| \cos (\theta) \\
& d_{y}=-A|N \times P|_{y}+\left(P_{y}\left(N_{x}^{2}+N_{y}^{2}\right)-P_{x} N_{x} N_{y}-P_{z} N_{y} N_{z}\right)|P| \cos (\theta) \\
& d_{z}=-A|N \times P|_{z}+\left(P_{z}\left(N_{x}^{2}+N_{y}^{2}\right)-P_{x} N_{x} N_{z}-P_{y} N_{y} N_{z}\right)|P| \cos (\theta)
\end{aligned}
$$

$$
\text { In this study, the rotation angle is fixed at } 90^{\circ} \text {, which is consistent with the previous studies }
$$

$[16,20,22]$. The branching angle $(\theta)$ has a significant effect on branch length. this prevents the growth of other airways and consequently, the development of conducting airways would be stopped before reaching the acinar region. Besides, this causes the terminal 
391 bronchioles with an inappropriate length to be generated. For example, an improper branching

392 pattern may lead to a bronchiole with a large diameter lies near the boundary, while its diameter

393 is not in the reasonable range. Furthermore, since it is in the vicinity of the boundary it cannot be

394 long enough and this causes too low length-to-diameter ratio. Such a scenario results in

395 inappropriate short branches. In addition, this may similarly cause small diameters. Furthermore,

396 the orientation of the branch affects the tree weight, fluid friction, and energy dissipation.

397 Therefore, it is essential to determine this parameter properly. Kitaoka[16] considered a simple

398 bifurcation and obtained Eq. (3.11) based on the minimization of power dissipation and the total

399 volume of bifurcation. Davoodi and Boozarjomehry [22] used Eq. (3.11) for this purpose.

$$
\begin{aligned}
& \cos \left(\theta_{1}\right)=\left(1+r^{4 / n}-(1-r)^{4 / n}\right) / 2 r^{2 / n} \\
& \cos \left(\theta_{2}\right)=\left(1+(1-r)^{4 / n}-(r)^{4 / n}\right) / 2(1-r)^{2 / n}
\end{aligned}
$$

400 However, all these methods were solely based on ongoing bifurcation, and this does not grantee

401 the minimization of the objective function for the whole structure of the human lung. Although

402 these mathematical relations decrease the resistance in the current bifurcation, it may result in lots

403 of bifurcations leading to the severe increase of total resistance. On the other hand, the human lung

404 is not physically optimized and such models are physiologically dangerous[32]. Therefore, an

405 intelligent procedure is required to lead the branches to the corresponding boundary so that the

406 anatomical constraints of the human lung are satisfied. Thus, instead of using any of the above

407 relations(e.g., Eq. (3.11)), the growth of each branch is examined in the several angles to obtain

408 enough information about the surrounding then the best angle is chosen based on some heuristic

409 rules used to satisfy anatomical and physiological constraints. For this purpose, the angle changes

410 by increment $\Delta \theta$ between $\theta_{\min }$ and $\theta_{\max }$ and an angle is randomly selected at each subinterval

$411\left(\left[\theta_{i}, \theta_{i}+\Delta \theta\right)\right)$ by a normal distribution with a mean of $\theta_{i}$ and a standard deviation of $\Delta \theta / 2$. After

412 that, branch orientation is calculated for each $\theta$ using Eq. (3.9) or (3.10). To find the best branch 
413 orientation among these potential directions, the following rules are used. It should be noted that

414 each direction has its diameter, length, and distance to the corresponding boundary whose

415 calculation algorithm mentioned previously.

416 Rule 1: if $d / d_{T B} \leq 1.2$ then the probability of being $T B$ in the next few generations (i.e. up to 417 three generations) is high, thus a direction with the minimum length is chosen. This rule 418 prevents the production of TB's with too small diameter because if the minimum length was not 419 chosen, then there would be numerous growth cycles (generations) from this branch and this would 420 lead to branches whose diameters are significantly lower than their corresponding anatomically 421 meaningful values.

422 Rule 2: if $1.2<d / d_{T B} \leq 1.5$ then the probability of being $T B$ in the next few generations is 423 medium, hence a direction with the minimum distance to the corresponding boundary is chosen.

424 This rule leads branches to the corresponding bronchopulmonary segment boundary and prevents 425 growing procedure stopped in the middle of the segment.

426 Rule 3: if the length of the selected direction is too small $(L / d<2)$, this direction is replaced by 427 another one that satisfies this condition and has the least length. This rule prevents the 428 production of branches with too small aspect ratio.

429 Rule 4: if the probability of being TB in a few next generations is low (i.e., neither of the above 430 rules is fired), the best direction is the one with the highest score defined in Eq. (3.12). This rule 431 leads branches to the less crowded regions with higher lengths and thus the congestion caused by 432 the physical presence of the surrounding branches is reduced and a structure with the minimum 433 number of bifurcations is well constructed. 


$$
\mathrm{S}=\frac{\mathrm{L}}{\mathrm{L}_{\max }}+\max ((\text { gen }-15), 1) \frac{\rho}{\rho_{\max }}
$$

434 where $\rho$ is a measure of emptiness around a growing branch which is defined in Eq. (3.13).

$$
\rho_{i}=\frac{\sum_{j=1}^{N} l_{i j}}{N}
$$

435 where $\mathrm{N}$ is the number of branches with non-zero collision probability around the growing branch.

$436 l_{i j}$ is the distance between the center of the $\mathrm{i}$ 'th branch and that of the $\mathrm{j}$ 'th branch. The second term 437 will be dominant in the last generations because of $\max (($ gen -15$), 1)$.

438 As mentioned above, these rules generally conduct branches to bronchopulmonary segments 439 boundaries and less crowded regions. These rules primarily may cause few branches to be 440 generated because they may direct the branches forward rather than distribute them. To solve this 441 problem, the initial range of the branching angle $\left(\theta_{\min }\right.$ and $\left.\theta_{\max }\right)$ should be determined based on 442 its relative distance to the corresponding boundary in the axial and lateral directions to provide the 443 branch with the necessary information about its vicinity. These parameters tell the branch that it 444 is in a round space or a long thin region. In addition, to ensure that the lung surface and the 445 segments' interfaces are fed at least by one branch, the adjacent bronchopulmonary segments are 446 also considered in the determination of the angle range. Hence, conducting airways in all segments 447 should be generated simultaneously. If a portion of the interface is not fed by branches in the 448 neighbor segment, the angle is set in such a way that the chance of getting it fed through the 449 growing branch increases. Accordingly, the ratio of the distance between a branch and its segment 450 interface to the segment diameter along the direction of branch orientation $\left(l d_{\text {axial }}\right)$, and the same 451 for the lateral direction $\left(l_{\text {lateral }}\right)$, and the congestion difference on both sides of the interface $(\Delta \beta)$ 
452 are applied to obtain the range of the branching angle. $\Delta \beta$ is defined in Eq. (3.14) and calculated 453 for each cell of the interface.

$$
\begin{aligned}
& \beta=\frac{\sum_{i=1}^{N} A_{i}}{A_{\text {cell }}} \\
& \Delta \beta=\beta_{\text {neighbor }}-\beta_{\text {owner }}
\end{aligned}
$$

454 where Acell and $\mathrm{A}_{\mathrm{i}}$ are cross-section area of a triangular boundary cell and its adjacent airways, 455 respectively.

456 Although we understand that the range of branching angle depends on the mentioned parameters,

457 the exact relation is unknown. However, we know qualitatively the effect of each parameter on the 458 branching angle. To find the branching angle based on non-numerical information, the fuzzy 459 inference system is employed. The fuzzy inference system is a type of inference systems in which 460 the reasoning is done based on the facts and concepts whose truths are subjective (and /or cannot 461 be validated firmly). The logic behind such reasoning differs from conventional logic. In Fuzzy 462 logic, a fact might be partly true or false, while this is also the case for a rule or logical expressions. 463 The truth values of rules, logical expressions, and facts vary from 0 to 1 . The design of a fuzzy 464 inference systems includes three steps: 1) Specifying the Fuzzy sets comprising the universe of 465 discourse of each variable (either input or output) used in the inference system, 2) Development 466 of rule base which consists of all the rules (coming from intuition and/or results obtained from 467 qualitative or quantitative phenomenological studies) based on which the fuzzy inputs get mapped 468 to fuzzy outputs 3)Selection of defuzzification method by which the fuzzy values of each fuzzy 469 output variable transformed back to a crisp numeric value. There are various useful resources 470 (including books and articles) that the interested readers can refer to among which reference [45] 471 is very informative. 
472 The cascade fuzzy inference system is used to find the range of branching angles based on 473 previously mentioned affecting parameters. Figure 4 shows a schematic of the fuzzy system.

474 Figures 4-7 indicate the fuzzy sets for the input and output variables and the rule table of the fuzzy 475 inference system, respectively. The rule table has been prepared based on our knowledge of the 476 structure. $l d_{\text {lateral }}, l d_{\text {axial }}$, and $\Delta \beta$ are divided into 3 fuzzy sets, and $\theta_{\mathrm{i}}, \theta_{\min }$, and $\theta_{\max }$ are divided 477 into 5 fuzzy sets. Symbols VL, L, M, H, VH, N, Z, P denote Very Low, Low, Medium, High, Very 478 High, Negative, Zero, and Positive, respectively. It should be noted that the first input of the second 479 fuzzy system will be $\theta_{\min }^{i}\left(\theta_{\max }^{i}\right)$ if its output is $\theta_{\min }^{\mathrm{i}}\left(\theta_{\max }^{\mathrm{i}}\right)$.

480

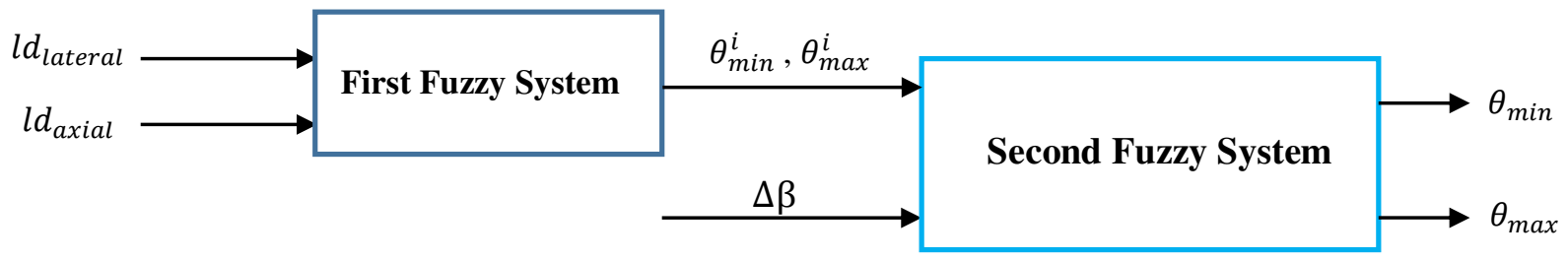

Fig. 4 Fuzzy logic system to find the range of branching angle

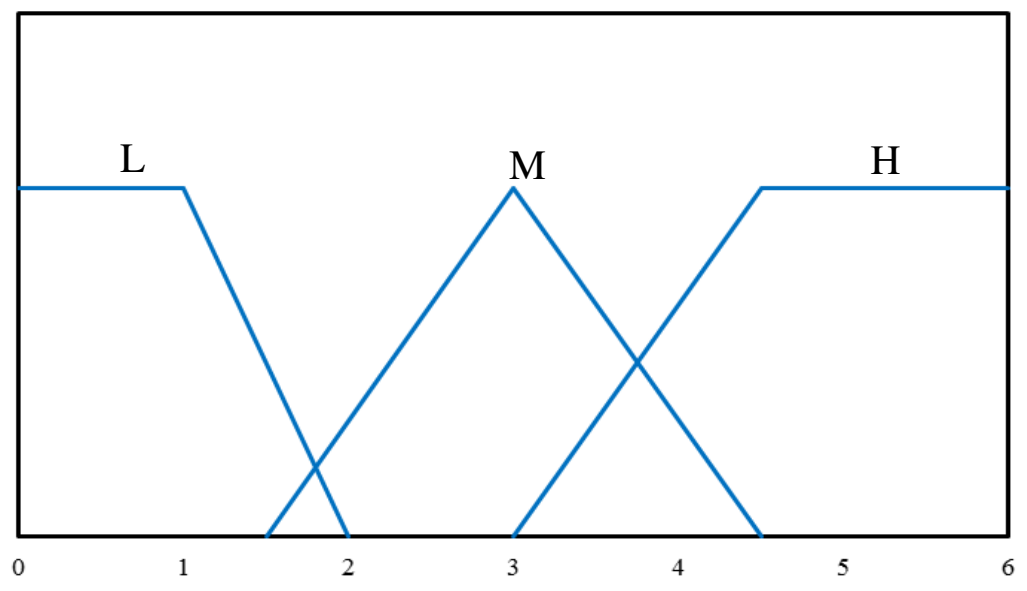

Fig. 5 Fuzzy sets for $\operatorname{ld}_{\text {lateral }}$ and $\operatorname{ld}_{\text {axial }}$ 


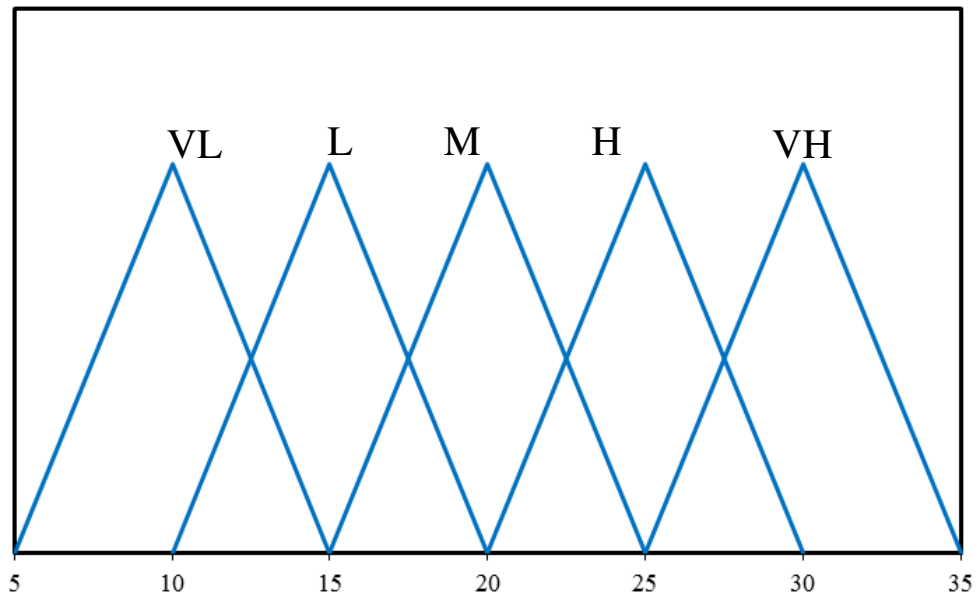

486

Fig. 6 Fuzzy sets for $\theta_{\min }$ and $\theta_{\min }^{\mathrm{i}}$

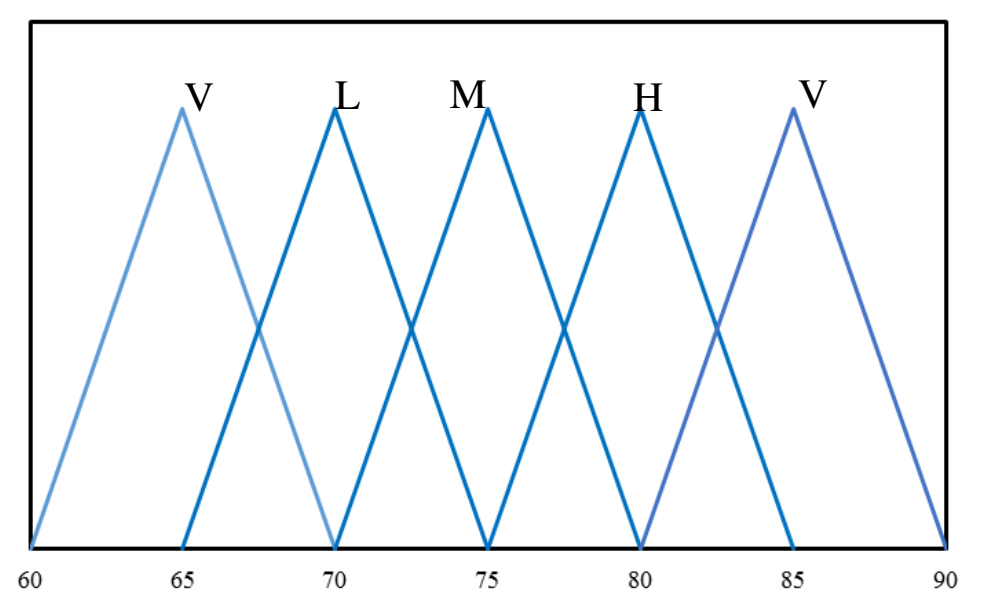

Fig. 7 Fuzzy sets for $\theta_{\max }$ and $\theta_{\max }^{\mathrm{i}}$

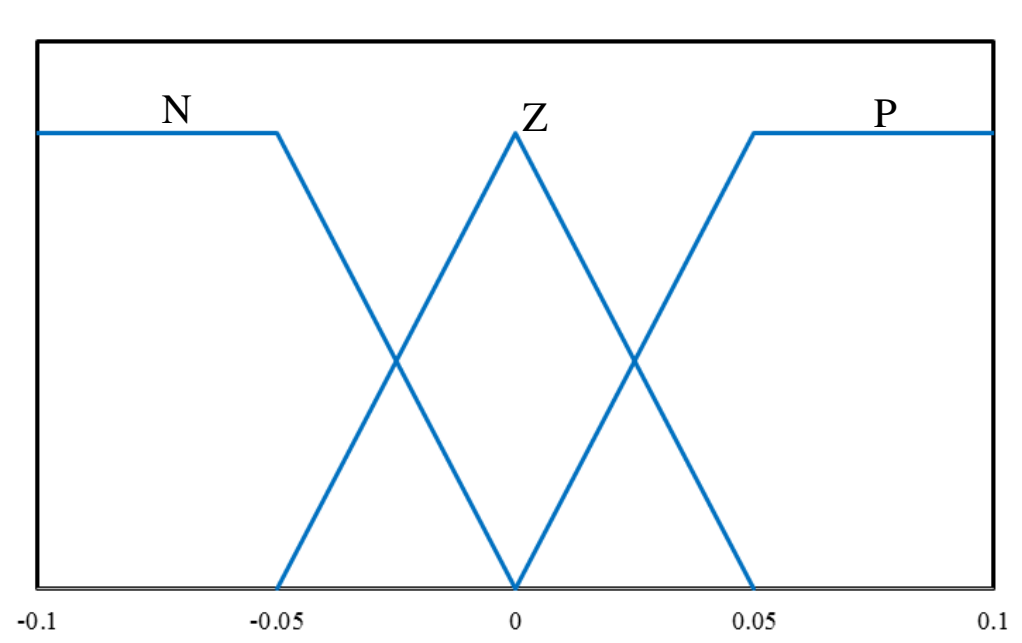

Fig. 8 Fuzzy sets for $\Delta \beta$ 
Table 4. Rules for the first fuzzy system

492

493

494

495 In the human bronchial tree, there are some branches called "links" whose orientations are in 496 the direction of their parent orientation. In order to include the links in the structure, the best

497 direction of each daughter is compared with the link and replaced via the following procedure. It

498 should be noted that only one daughter can be replaced and the procedure determines which one 499 is compared with the link. It worth noting that the "links" have been ignored in model of Davoodi 500 and Bozorgmehry[22].

i) If $\theta_{1}\left(\theta_{2}\right)=\hat{\theta}_{\text {min }}$ and $\theta_{2}\left(\theta_{1}\right)>\hat{\theta}_{\text {min }}$, the daughter 1(2) is compared with the link to prevent 502 a very small angle between daughters.

503

504

505 links are avoided.

506

According to the production rules, each branch is divided into two daughter branches provided

507 that the parameters of the daughters are determined. The orientation and dimensions of the 508 daughters are found via the following steps: 
509 1. A random value is chosen for ' $r$ ' from a random number generator with a uniform 510 distribution.

5112 . The diameter of each branch is calculated using the diameter ratio given in Eq. (3.4).

512 3. Using a predefined value for rotation angle, $\varphi$, the normal vector of daughters' plan $513 \quad$ calculated by Eq. (3.7).

514 4. The lateral and axial distances of each branch to the host boundary are calculated.

515 5. The value of parameter $\Delta \beta$ is obtained using Eq. (3.14).

516 6. The range of branching angle $\left(\theta_{\min }\right.$ and $\left.\theta_{\max }\right)$ are determined through the proposed fuzzy $517 \quad$ scheme.

518 7. Several branching angles are selected in the range of $\left(\theta_{\min }\right.$ and $\left.\theta_{\max }\right)$, and the branch 519 orientation corresponding to each branching angle is calculated using Eq. (3.9) and Eq. $520 \quad(3.10)$.

5218 . The distance to the boundary and parameter D is calculated for each potential branch 522 orientation. Then the corresponding branch length is calculated by Eq. (3.5).

523 9. The best orientation of each branch is selected among the potential orientations through the $524 \quad$ proposed predictive rules.

525 10. At most one daughter branch is compared with the link and the best direction is chosen $526 \quad$ using the same predictive rules.

527 11. The daughters are compared and if the branch with a larger diameter does not have a greater 528 length, the procedure is repeated from step 1. Because the branch with greater length has a 529 greater distance to the boundary and is located in the less crowded region, it should be 530 thicker to deliver air to the larger volume. 
12. Although the predictive rules are designed to find the best direction, there may be few terminal bronchioles with too small length. To remove such branches in the structure, the algorithm goes back and reduces the diameter ratio in order for the paths to reach to terminal bronchioles earlier and stop their growths in this direction before too short branches are produced.

538 the children. Menache et.al [46] proposed an equation for the diameter of terminal bronchioles of 539 subjects aged 0.75 yrs to $30 y$ rs. To find $d_{T B, \max }$, we improved the equation by multiplying it in a 540 correction facrtor so that it gives $d_{T B, \max }$ of $0.8 \mathrm{~mm}$ for adults. The equation is given in Eq. (3.16).

541 The value of $L_{T B, \max }$ is assumed to be equal to $2.25 d_{T B, \max }$.

$$
d_{T B, \max }=0.9236-0.7547 e^{-0.0603 \text { age }(y r s)}
$$

\section{Results}

544 In this section, the segmentation results of bronchopulmonary segments are presented. Then the

545 generated bronchial tree using the proposed method is evaluated through comparison of 546 geometrical properties with morphological measurements and the other alternate methods. The 547 analyses are performed for both central airways and the whole structure. In what follows, the 548 daughter branches with the smaller and larger diameters are called the minor and major branches, 549 respectively. The subscripts 'min', 'maj', and 'p' denote minor, major, and parent branches, 550 respectively. The results correspond to a total lung capacity of 5500ml. 

The central airways have been generated via three generations of applying production rules.

552 The mean diameter of segmental bronchi, lobar bronchi, and total airways are $4.32 \mathrm{~mm}, 7.8 \mathrm{~mm}$, 553 and $6.59 \mathrm{~mm}$ which is comparable with the value of 4.61, 6.73, and 6.29 measured by Horsfield 554 et.al [13]. The insignificant difference between the values may originate from the difference in the 555 method of measurement and size of the lung. The geometric properties of central airways are 556 summarized in Table 6 . The mean values of $l / d, d / d_{p}$ and $d_{m i n} / d_{m a j}$ are consistent with the 557 previously reported values. The value of $l / l_{p}$ has large standard deviation because in upper airways 558 there are some branches with short parent length. The difference between the reported values 559 originates from the fact that they are based on various number of extracted airways and the 560 variations that exist in the structure of central airways among various people. Peripheral airways 561 are classified as Strahler order and Horsfield order of 1. The mean value of diameter for Strahler 562 order of 1,2 and 3 are $4.32 \mathrm{~mm}, 7.00 \mathrm{~mm}$, and $12.19 \mathrm{~mm}$, respectively. The results show the increase 563 of diameter with the Strahler order. The variation of number, diameter, and length against the 564 Horsfield order are shown in Fig. 9. This figure indicates that the length doesn't decrease as 565 uniformly as diameter. The trend reveals that the length reduction has almost a linear trend for 566 small Horsfield orders.

Table 6. Geometric properties of central airways

\begin{tabular}{clccc}
\hline & \multicolumn{3}{c}{ Published values } \\
\cline { 2 - 5 } & The present method & {$[13]$} & \multicolumn{1}{c}{$[19]$} & {$[22]$} \\
\hline$l / d$ & $2.74 \pm 1.39$ & $2.15 \pm 1.16$ & $3.04 \pm 2.2$ & $3.42 \pm 1.61$ \\
$d / d_{p}$ & $0.70 \pm 0.14$ & $0.75 \pm 0.16$ & $0.71 \pm 0.14$ & $0.63 \pm 0.16$ \\
$l / l_{p}$ & $0.98 \pm 0.61$ & $1.10 \pm 1.30$ & $1.18 \pm 1.1$ & \\
$d_{\text {min }} / d_{p}$ & $0.60 \pm 0.1$ & $0.67 \pm 0.08$ & $0.66 \pm 0.12$ & \\
$d_{\text {maj }} / d_{p}$ & $0.79 \pm 0.09$ & $0.83 \pm 0.17$ & $0.79 \pm 0.12$ & \\
$d_{\text {min }} / d_{\text {maj }}$ & $0.78 \pm 0.17$ & $0.83 \pm 0.13$ & $0.85 \pm 0.14$ & $0.69 \pm 0.17$ \\
$\theta$ & $41.6^{\circ} \pm 17.5^{\circ}$ & & $36.11^{\circ} \pm 20.85^{\circ}$ & $37.38^{\circ} \pm 15.75^{\circ}$ \\
\hline
\end{tabular}




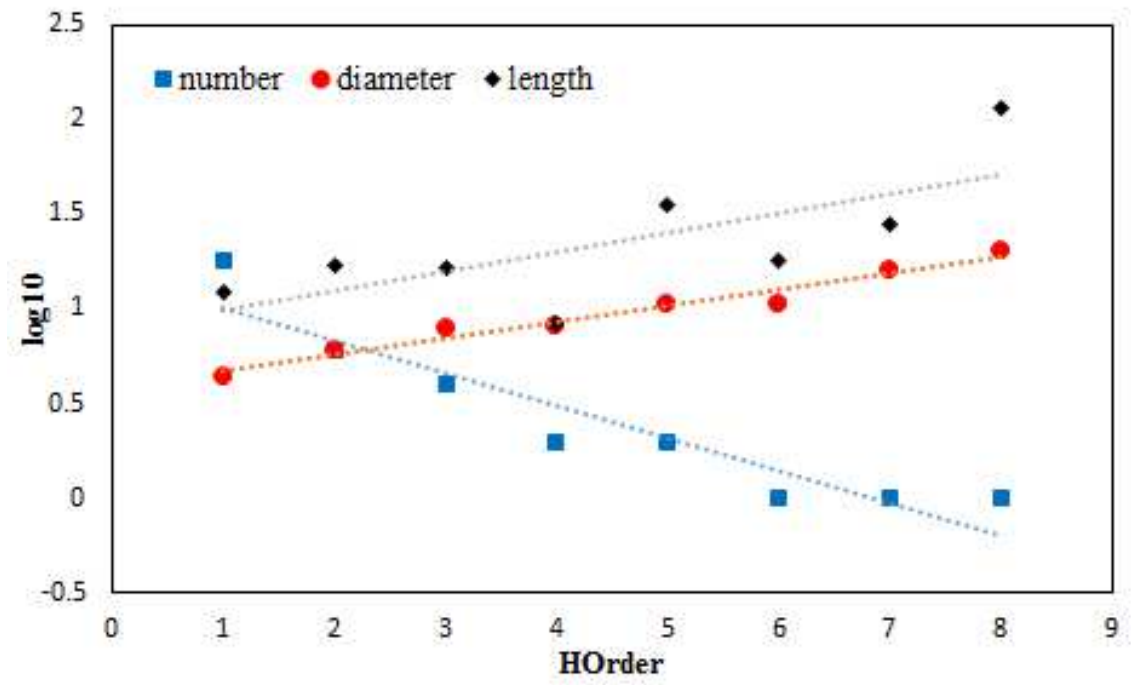

Fig. 9 Variation of the number of branches, mean diameter and mean length of central airways against Horsfield

571 The bronchopulmonary segments were obtained using 3DSlicer software. The segmentation

572 results for both right and left lungs are shown in Figs. 10-11. The eighteen dichotomous structures

573 are merged with the central airways to generate the whole structure of the tracheobronchial tree as

574 shown in Fig. 12. Each bronchopulmonary segment and central airway are shown with different 575 colors: central airways are gray and the color of each segment is similar to its corresponding ones

576 in Figs. 10-11. In addition, bronchial airway tree produced by parametric L-system for the smallest 577 segment (RL_medial), and two of the largest segments (RL_posterior and LU_anterior) are 578 indicated in Figs. 13-15. These figures indicate that the bronchial tree has grown properly up to 579 the host boundaries. 

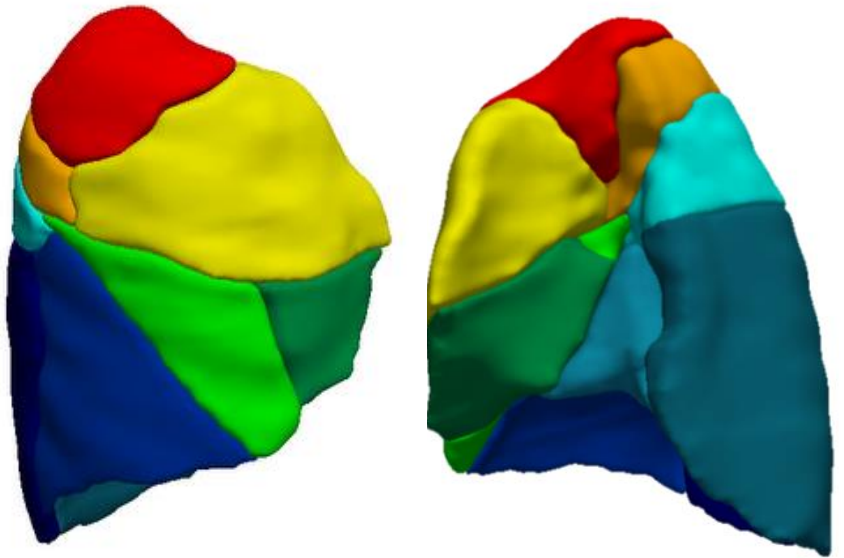

Fig. 10 Bronchopulmonary segments of the right lung
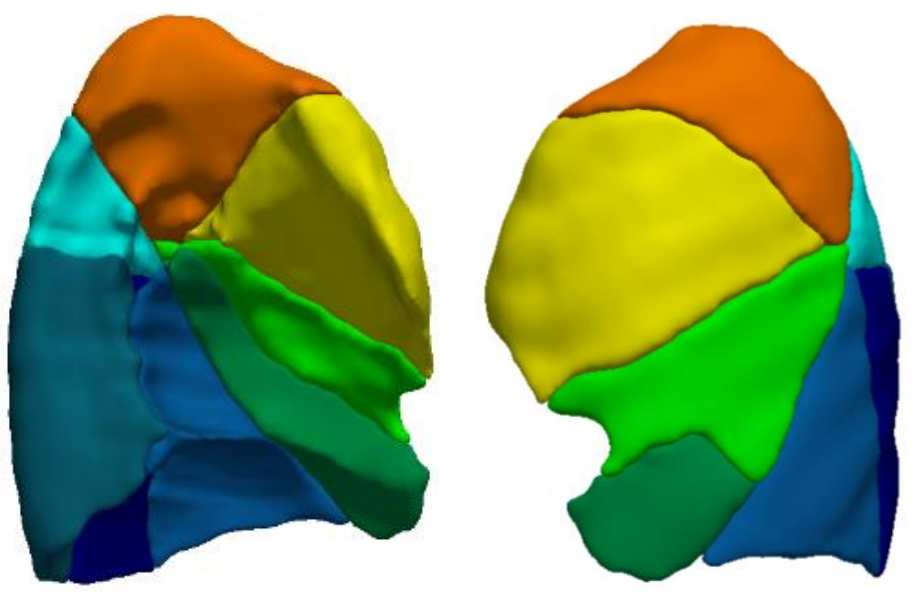

Fig. 11 Bronchopulmonary segments of the left lung.

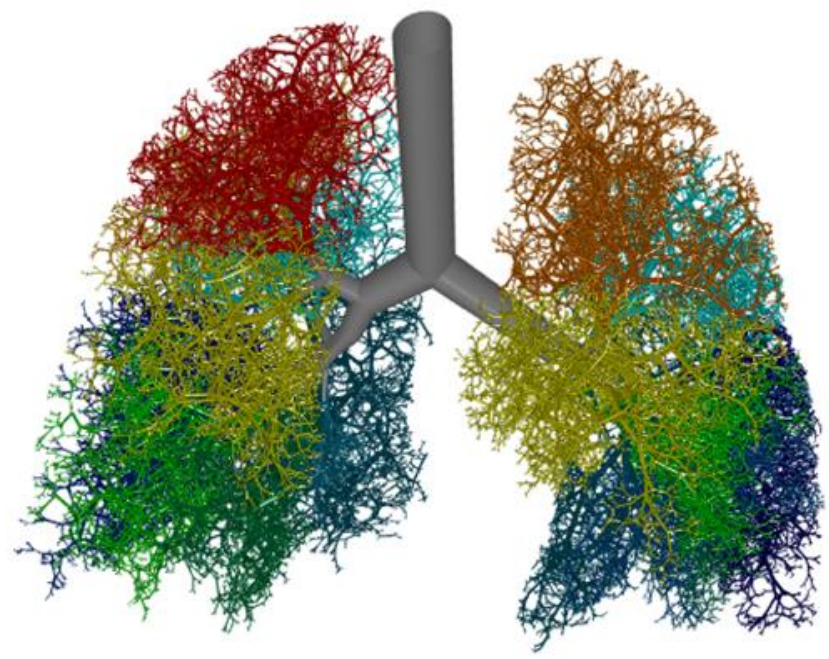

Fig. 12 Anterior view of conducting airway produced by the proposed method 
586

587

588
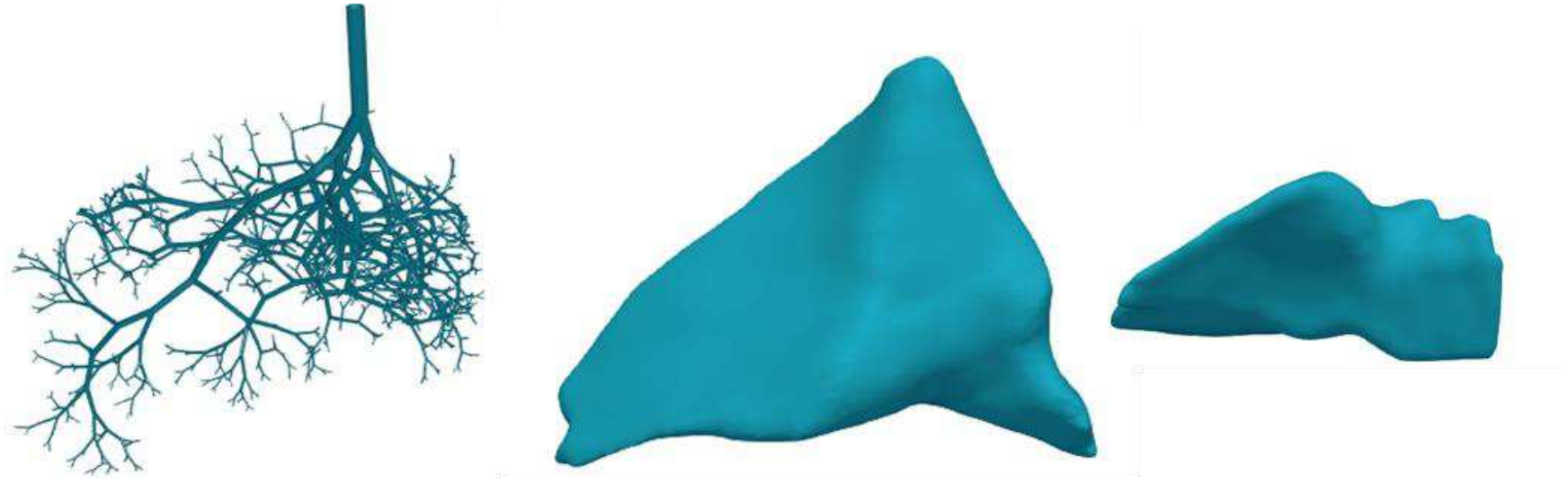

Fig. 13 Conducting airways in RL_medial produced by the proposed method
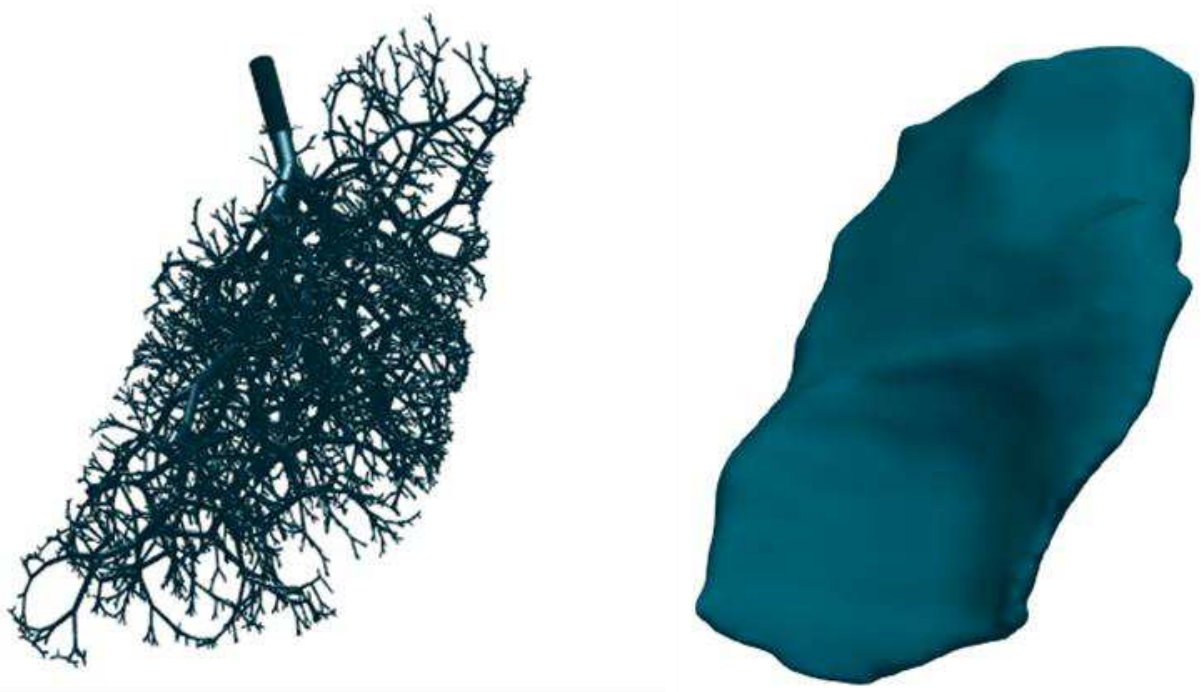

Fig. 14 Conducting airways in RL_Posterior produced by the proposed method
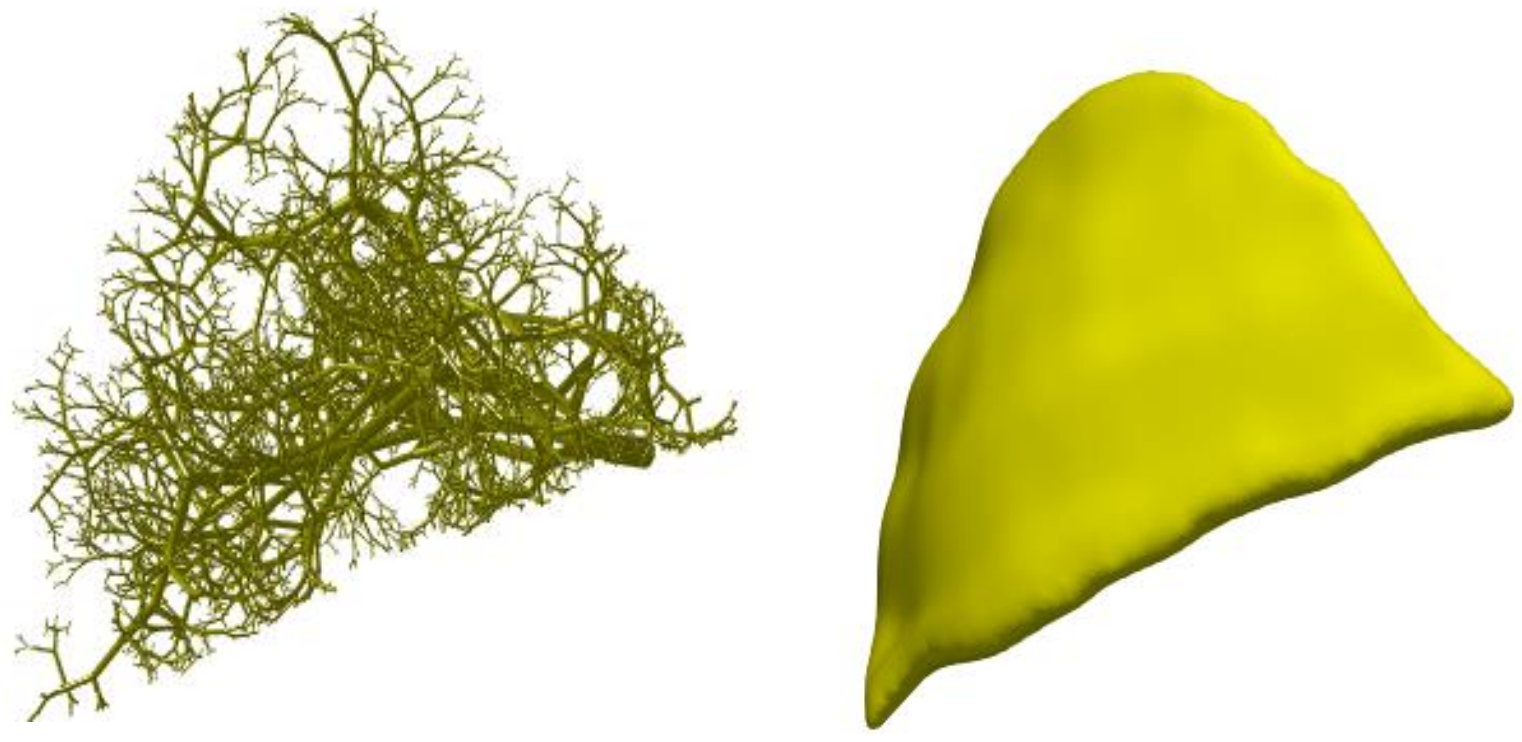
593 The generation number is a measure of the distance of a branch and the trachea. The greater the 594 generation number reflects that the branches are further from trachea. Table 7 indicates the mean, 595 minimum and maximum generation number of terminal bronchioles in the lungs and each lobe.

596 The acini are appeared in the mean generation of 16.7, with a minimum of 8 and maximum of 25.

597 The number of TB's in the structure obtained by the proposed method is 30201 and is comparable

598 with those reported in the literature as shown in Table 7. The number of TB's obtained by Haefeli-

599 Bleuer and Weibel [23] lies in the range of 26000 to 32000 . Generation number of TB's is a 600 measure of the number of bifurcations from trachea at which an acinus may appear. Lobes that are

601 further away from trachea have a larger mean generation number of TB's as shown in Table 7. For 602 instance, the right lower lobe has the largest generation number because it has the furthest distance 603 from the trachea and is the largest lobe.

Table 7. Number of terminal bronchioles and corresponding generation number

\begin{tabular}{|c|c|c|c|c|c|c|c|c|c|}
\hline & \multirow[t]{2}{*}{ NO of Acini } & \multicolumn{8}{|c|}{ Generation distance of acini from the trachea } \\
\hline & & RU & $\mathrm{RM}$ & $\mathrm{RL}$ & LU & LL & Lungs & Min & $\max$ \\
\hline The present method & 30201 & 15.9 & 16.2 & 18.3 & 16.5 & 16.6 & 16.7 & 8 & 25 \\
\hline$[21]$ & 27763 & 15.3 & 15.2 & 17.2 & 15.9 & 16.3 & $16.2 \pm 1.7$ & 8 & 25 \\
\hline$[10]$ & & & & & & & 15 & 8 & 25 \\
\hline [16] & 28313 & & & & & & $17.6 \pm 3.4$ & 8 & 32 \\
\hline [47] & & 15 & 15 & 17 & 15 & 16 & 15.6 & & \\
\hline [18] & 29445 & 16.4 & 16 & 17.4 & 16.3 & 16.2 & 16 & 10 & 26 \\
\hline
\end{tabular}

605

606 Although using generation number is useful for locating the branches in relation to the trachea,

607 it is not appropriate for asymmetric trees because this classification locates very dissimilar 608 branches in one generation. Therefore, the branches should be classified by order instead of 609 generation. Strahler and Horsfield orders are the most common classifying methods for 610 asymmetric trees. The number of branches is decreased with the increase in Strahler and Horsfield 
611 orders and hence trachea has the highest Strahler and Horsfield orders. The relative increase in

612 each order is called branching ratio, $\mathrm{Rb}$, and is defined as the antilog of the slope of the line

613 representing the number of branches versus the branching order in a logarithmic plot. The diameter

614 ratio $(\mathrm{Rd})$ and length ratio $(\mathrm{Rl})$ are calculated similarly and are measures of the reductions in

615 diameter and length, respectively. Figures 16-17 show the logarithmic plot of the number of

616 branches, diameter, and length against Horsfield and Strahler orders. Table 8 compares Rb, Rd,

617 and Rl obtained by the proposed method and their corresponding published values. The value in

618 parenthesis is R squared (coefficient of determination) of the corresponding variable. Prefixes "S"

619 and " $\mathrm{H}$ " indicate the parameters calculated from Strahler and Horsfield ordering, respectively.

$620 \mathrm{HRb}$ and $\mathrm{SRb}$ are equal to 2 for symmetric trees. SRb becomes greater than 2 and $\mathrm{HRb}$ approaches

621 to 1 with an increase in asymmetry. SRb of 2.76 obtained in this study is higher than those given

622 by $[18,21,47]$ and less than those predicted by $[19,22]$. HRb of 1.52 is consistent with the

623 published values. The difference in the reported values may be originated from the degree of

624 asymmetry of the methods and the shape of the lungs. Round lungs have a lower Rb than long thin

625 ones. Figure 15 shows a more linear trend comparing to those that exist in Fig. 16 and hence SRb

626 is more consistent throughout the lungs. However, every branch is not separately ordered in

627 Strahler ordering method. This makes a simpler description with loss of details compared to the

628 Horsfield ordering method in which each branch is separately ordered. To minimize resistance or

629 entropy production, the value of $\mathrm{Rd}$ is expected to be almost equal to $\mathrm{Rb} 1 / 3$ [48]. The predicted

630 values for $\mathrm{Rb}$ and $\mathrm{Rd}$ well follow this relation (1.12\% relative deviation for Strahler ordering and

$631 \quad 0.25 \%$ for Horsfield ordering). 


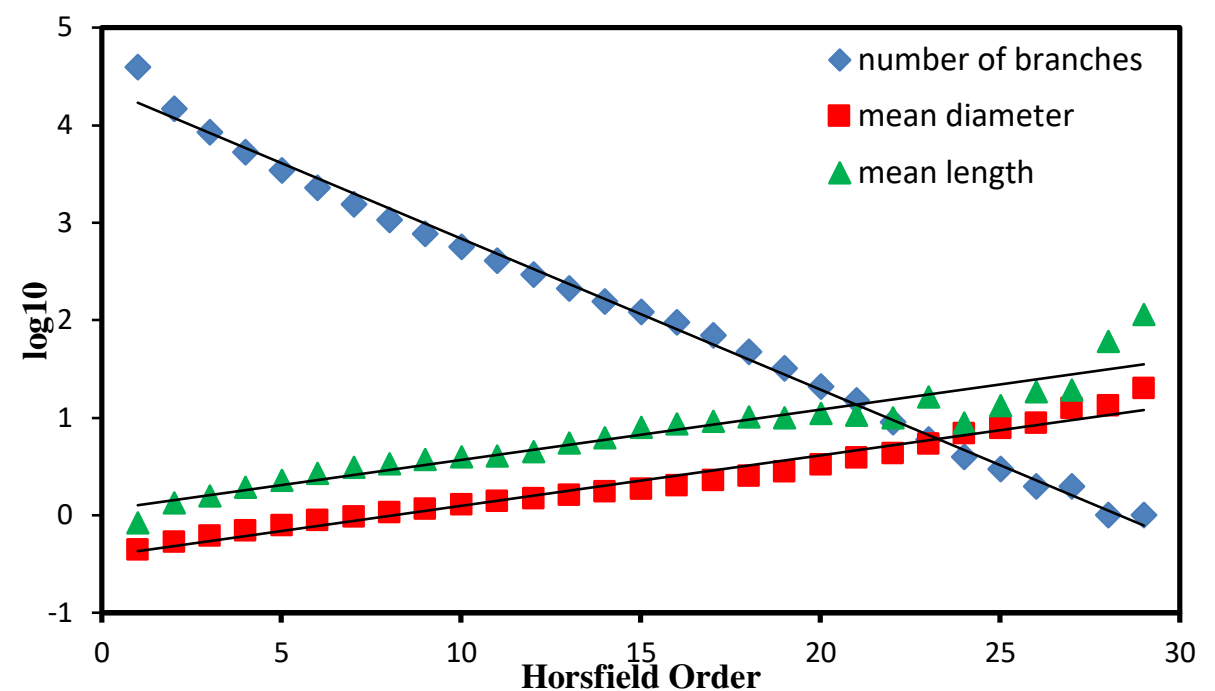

Fig. 16 Number, length, and diameter of airways against Horsfield order

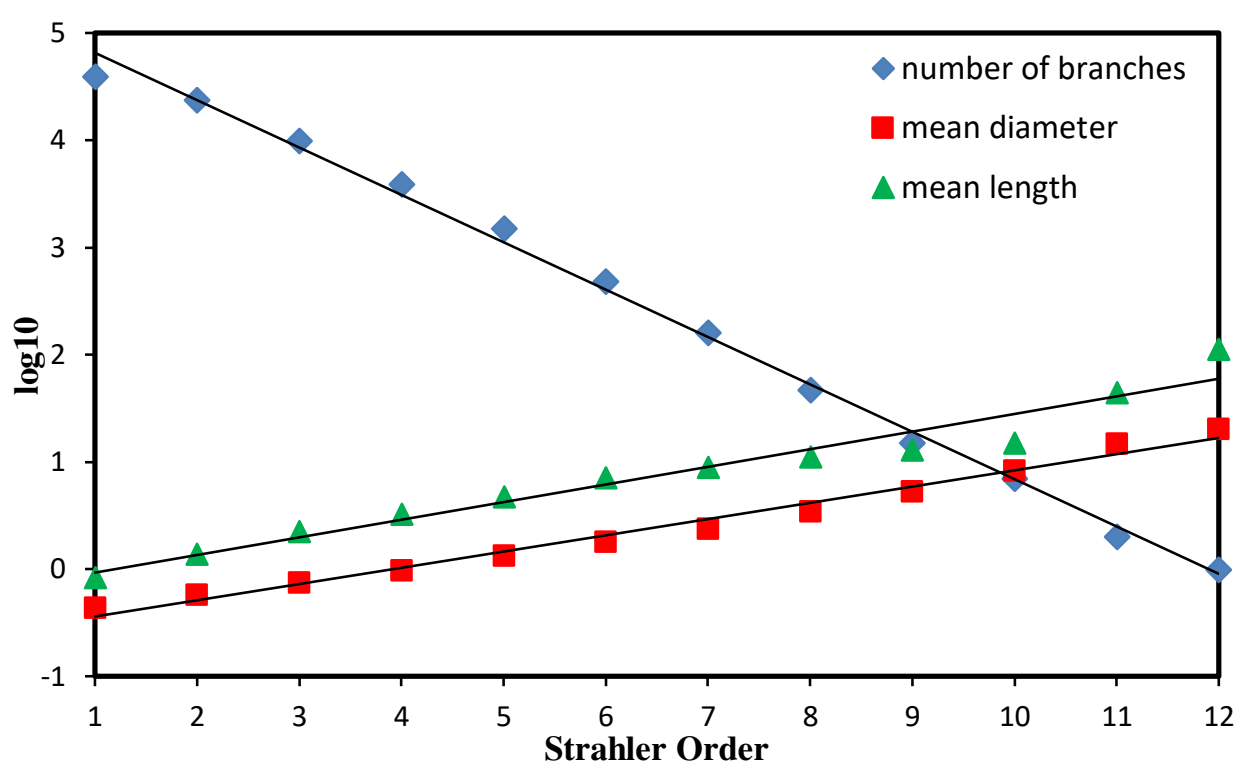

Fig. 17 Number, length, and diameter of airways against Strahler order

636 Table 8. Structural properties of the human bronchial tree obtained by the proposed method and previous studies

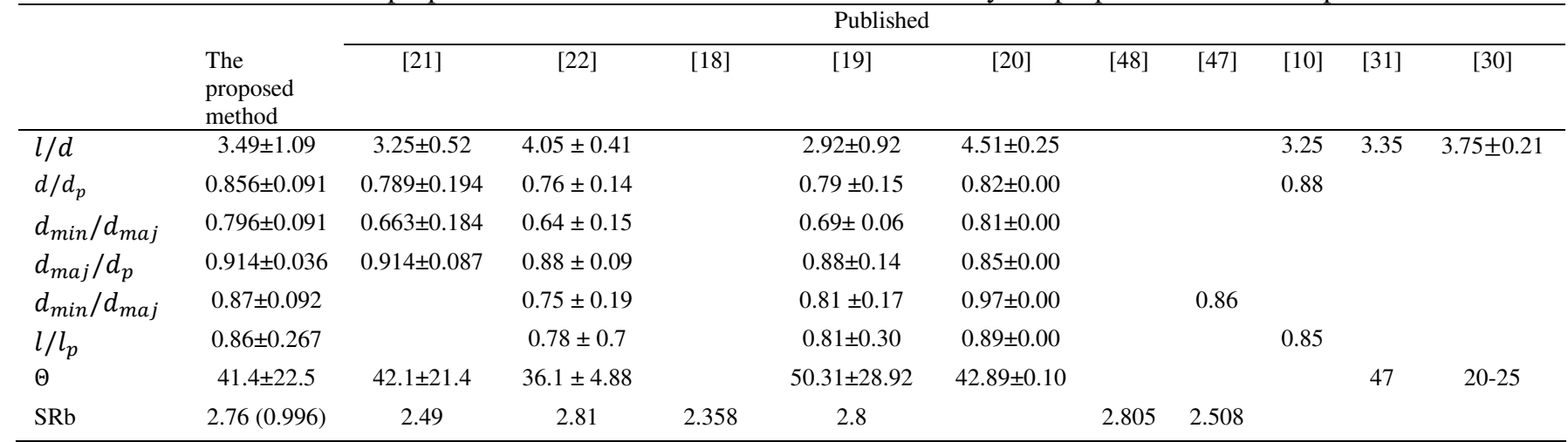




\begin{tabular}{lccccccc}
\hline SRd & $1.42(0.99)$ & 1.397 & 1.51 & 1.323 & 1.41 & 1.427 & 1.351 \\
SRl & $1.40(0.95)$ & 1.392 & 1.47 & 1.344 & 1.39 & 1.402 & 1.33 \\
HRb & $1.51(0.99)$ & 1.56 & 1.44 & 1.39 & 1.47 & & \\
HRd & $1.15(0.97)$ & 1.166 & 1.14 & 1.109 & & \\
HRl & $1.12(0.89)$ & 1.13 & 1.11 & 1.11 & 1.13 & \\
\hline
\end{tabular}

637

638 Table 8 compares the geometrical properties of the tracheobronchial tree generated in this study

639 with those reported in the previous studies.

640 Because the diameters of three branches involving in a bifurcation are different, it is of interest

641 to observe the relation between them. In this study, the diameter decreased based on Eq. (3.4)

642 reflecting the relationship between flow rate and branch diameter in a living organ to make it

643 capable of overcoming the energy lost due to fluid friction in an optimal manner. For typical human

644 bronchial tree the diameter exponent, ' $n$ ', is approximately $2.9_{2}$ [48], which is closer to that in the

645 laminar flow. We examined several values for ' $n$ ' in the range of 2.4 to 3.5 and found that the best

646 structure could be obtained with a value of 2.8 as proposed by Kitaoka et al[16] and used by

647 Davoodi and Bozorgmehry[22]. Weibel [29] showed that D/Dp is higher for generations $>10$ with

648 a minimum average value of 0.84 . Therefore, the minimum value of 0.7 was considered for $\mathrm{D} / \mathrm{Dp}$

649 in generations $>10$. The stochastic behavior of the proposed procedure originates from the random

650 flow-dividing ratio (r) building slightly different structures with the same structural properties. It

651 is randomly set for each branch in such a way that the larger host volume is fed by the major

652 daughter $r . \mathrm{D} / \mathrm{D}_{\mathrm{p}}, \mathrm{D}_{\min } / \mathrm{D}_{\mathrm{p}}, \mathrm{D}_{\mathrm{maj}} / \mathrm{D}_{\mathrm{p}}, \mathrm{D}_{\mathrm{min}} / \mathrm{D}_{\text {maj }}$ are consistent with those reported in the previous

653 studies. The obtained value of $\mathrm{D} / \mathrm{D}_{\mathrm{p}}$ is 0.856 which is higher than that predicted by the previous

654 methods[19-22] and slightly less than that measured by Weibel [29]. In an ideal fractal tree, this

655 ratio is $0.79(21 / 3)$. However, Mauroy et al [32]showed that the human bronchial tree is not

2 It is approximated as $\log (\mathrm{SRb}=2.805) / \log (\mathrm{SRd}=1.427)=2.9$ 
656 physically optimal and the ratio in the real tree is about 0.85 which is approximately equal to that

657 obtained in this study. Diameter reduction in the human bronchial tree is greater than that predicted

658 by the previous models and that is due to the fact that the physiological robustness of human lung

659 is obtained at the expense of its deviation from the physically optimum structure. This

660 physiological robustness makes the lungs functional in the case of slight damage in its structure.

661 That is, the diameter ratio in the proposed method is greater than that in the previous models.

662 Furthermore, D/Dp predicted by the proposed method has been compared with those measured by

663 Weibel[29] and Yeh and Schum[11] in Fig. 18. This figure confirms the ability of the proposed

664 procedure in the prediction of real data. $d_{\min } / d_{m a j}$ calculated by the present model is much smaller

665 than those reported by Bordas et.al [20] because the present method is more asymmetric comparing

666 to their obtained structure[20]. It is greater than those reported in [22] and [19] and is comparable

667 to [47]. Weibel [29] measured $d_{m i n} / d_{m a j}$ of $0.86 \pm 0.01$, which is almost the same as the value 668 predicted in our work.

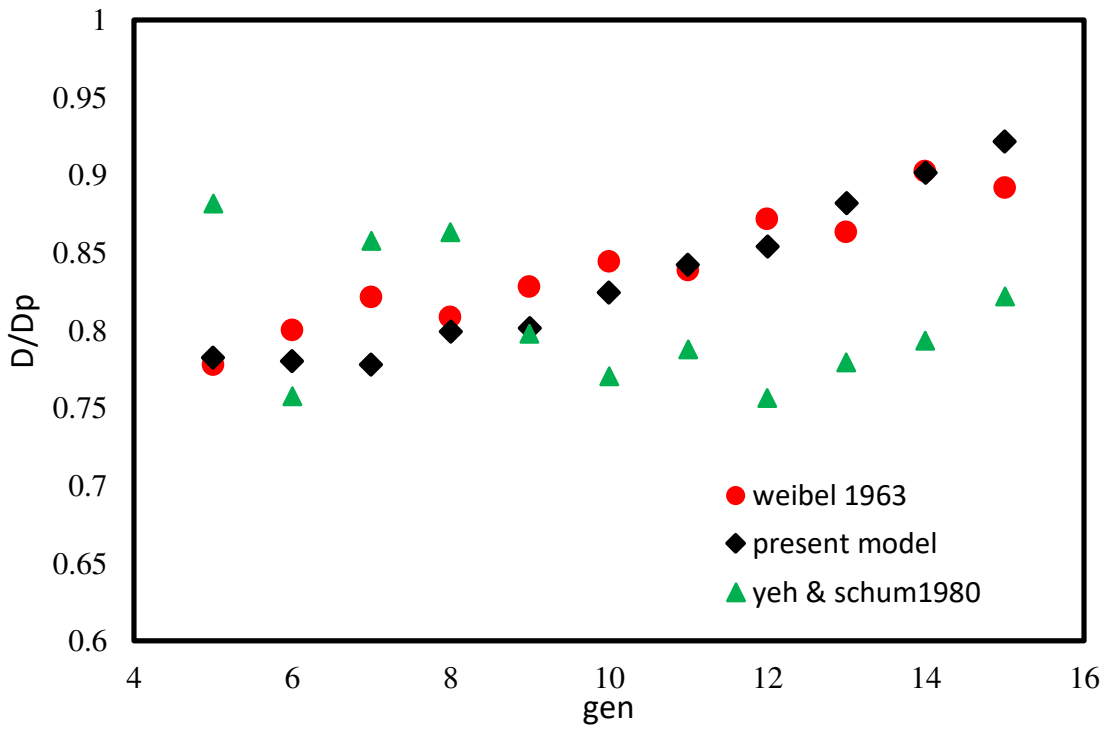

Fig. 18 Diameter ratio against generation number 
672 The predicted mean value of the length-to-diameter ratio is inline with those calculated in the

673 previous studies as indicated in Table 8. The higher standard deviation (SD) may be originated

674 from considering bronchopulmonary segments as host volume while the tree was grown in the

675 lungs or lobes in the previous studies. For instance, the inferior lingular is a long thin segment.

676 Therefore, airways along axial direction should have a large length, whereas those in lateral

677 direction have smaller length, this results in greater standard deviation compared to the previous

678 models. However, the coefficient of variation is comparable to what estimated by Tawhai et.al 679 [19].

$680 l / l_{p}$ has a higher standard deviation than $d / d_{p}$ because the length doesn't decrease as uniformly 681 as diameter. Figures 16-17 also confirm the result where the trend of diameter reduction is more 682 linear than that of length.

683 The present method predicts TB's diameters (Dтв) of $0.49 \pm 0.065 \mathrm{~mm}$. The previous clinical 684 studies measured Dтв of $0.432 \pm 0.035 \mathrm{~mm}$ [39] and $0.5 \pm 0.054 \mathrm{~mm}$ [23]using direct cast analysis, 685 and $0.66 \pm 0.04 \mathrm{~mm}$ [49] using synchrotron CT in a limited number of images. Previous models 686 predicted Dтв using some particular stopping conditions. Dтв of $0.48 \pm 0.06 \mathrm{~mm}, 0.375 \mathrm{~mm}, 0.6 \mathrm{~mm}$, 687 and $0.418 \pm 0.114 \mathrm{~mm}$ reported by [16], [18], [19], and [20], respectively. Although Davoodi and 688 Bozorgemhry [22] did not report the value of Dтв, we obtained too small TB's using their method. 689 This is a major shortcoming because the small dimensions of TB's consequently affect the size of 690 the acini fed by them and subsequently underestimate the lung volume and gas exchange area too 691 much.

692 The TB length (Lтв) of $0.87 \pm 0.40 \mathrm{~mm}$ has been predicted by the proposed method. The Lтв of $6930.819 \pm 0.14 \mathrm{~mm}, 0.8 \pm 0.354 \mathrm{~mm}$, and $0.93 \pm 0.43 \mathrm{~mm}$ reported by [39], [23], and [40] using the 
694 direct measurements on TB's. However, the values of $1.34 \pm 0.44 \mathrm{~mm}$ and $\sim 1.8 \mathrm{~mm}$ predicted by

695 [20] and most of the previous models [19, 31, 50]. The Lтв predicted by the present method is less

696 than those reported by the previous models and is more consistent with the experimental data. This

697 is because, in the present method, the airways grow inside the bronchopulmonary segments which

698 restrict the growth and length of TB's more than what lobes or lungs do.

699 The mean branching angle in our model is $41.4^{\circ}$. The mean branching angle is about $47^{\circ}$ based

700 on the data of 20 lung casts [31] and $41.26^{\circ}$ reported by [41] which is close to that calculated by

701 the proposed method. The calculated SD based on the data of [41] is $23.26^{\circ}$, which is almost the

702 same as what obtained in this study. Due to the asymmetric behavior of the tracheobronchial tree,

703 all proposed models experience relatively large standard deviations except the model of [20] with

704 almost no SD. Although the model of [20] has an asymmetric manner, it seems that the asymmetric

705 pattern is almost identical in all bifurcations as obvious from reported SD for other properties.

706 The calculated volume of the tracheobronchial tree is $133 \mathrm{ml}$. The volume of $145 \mathrm{ml}$ was measured

707 by Weibel et.al [29]. Kitaoka et.al [16]and Ismail et.al [51] estimated the volume of $175 \mathrm{ml}$ and

708 132.9ml, respectively. The difference between the reported values may correspond to the fact that

709 they are obtained based on the lungs of different subjects. The anatomical dead space measured by

710 the Fowler method is $156 \pm 28 \mathrm{ml}$. excluding the mouth volume of $40 \mathrm{ml}$ (estimated as $70 \%$ of the

711 maximum volume of mouth[52]), the volume of conducting airways is expected to be $116 \pm 28 \mathrm{ml}$.

712 Therefore, the value obtained by the proposed method is reasonable. The percentage volume of

713 airways in each lobe is shown in Table 9. This table indicates that the volume of conducting

714 airways is related to the volume of the corresponding lobe. 
Table 9. The percentage volume of conducting airways in each lobe

\begin{tabular}{cccccc}
\hline & RU & RM & RL & LU & LL \\
\hline Present Model & 18.0 & 8.7 & 25.7 & 22.7 & 24.9 \\
{$[18]$} & 22.8 & 10.2 & 22.5 & 23.7 & 20.8 \\
{$[12]$} & 21 & 9 & 25 & 20 & 25 \\
{$[51]$} & 16.0 & 9.2 & 27.4 & 24.9 & 22.5 \\
\hline
\end{tabular}

\section{Discussion}

720 In this study, to generate the human tracheobronchial tree, we tried to provide a robust rule-based

721 method whose parameters were determined using an intelligent procedure. The bronchial tree was

722 produced such that its various parts fit into bronchopulmonary segments obtained by analysis of

723 CT images and anatomical slices. Various parts of the tree obtained by the proposed algorithm are

724 completely contained by their corresponding host volumes in a homogenous manner while

725 fulfilling various statistical indices reported by anatomical and physiological research articles.

726 The spatial hindrance during growth of an airway due to the presence of surrounding airways

727 have been included in three-dimensional manner in this study, whereas it corresponding

728 counterpart was handled in one-dimensional in the previous works. Furthermore, the proposed

729 method predicted the more accurate dimensions of $\mathrm{TBs}$ and $\mathrm{D} / \mathrm{D}_{\mathrm{p}}$ ratio than their counterparts

730 obtained by previous asymmetric models.

731 In the current study, the TB's are distributed near the bronchopulmonary segments, which is

732 what happens in reality. Longitudinal pathways, the distances along the airways from the trachea

733 to TB's, have a great contribution to the resistance against the fluid flow and subsequently on the

734 ventilation and concentration distribution. Because TB's have been located near the boundaries of

735 lungs or lobes in the previous studies, the values of Longitudinal pathways should have a 736 significant deviation from reality but the values have not been reported. 
737 To construct the conducting airways in host volume, it is usually required to describe the surface

738 of the host volume, no matter whether the host volume is the whole lung, its constituting lobes, or

739 bronchopulmonary segments. Therefore, several methods have been used in the previous studies

740 for surface reconstruction: 1) thin-plate spline method, 2) surface fitting using hermit basis

741 function, 3) combination of some convex geometrical shapes. The latter has the least accuracy

742 among the others. On the other hand, the other two methods suffer from the fact that they are not

743 capable of representing all concave surfaces very accurately. The mathematical description of the

744 surface has not been used in the seed points-based methods, and it has been assumed that the new

745 branch is inside if the end position of the branch lies close enough to the grid points. Thus, although

746 the end of the branch lies inside the corresponding closed surface, it may intersect the boundaries

747 in at least two points due to the concaveness of the surface. Therefore, we used neither

748 mathematical relations for modeling of the surface nor grid points inside the host volume; instead,

749 the closed surfaces were described using a set of triangular cells and the ray-triangle intersection

750 algorithm was employed to find the distance to the boundary. This procedure overcomes the

751 mentioned limitations and can accurately describe various shapes of the closed surfaces.

752 In the proposed method, the rules of finding branching angle implicitly restrict branches to reach

753 back near one of their ancestors. In other words, the branches only go back if the desired region is

754 not fed by another branch that has a shorter pathway from the trachea. None of the alternative

755 methods has been addressing such an issue. This shortcoming may cause a more heterogeneous

756 flow distribution in the models than in the real lungs.

757 The major advantages of the proposed method are the following features that make the method 758 flexible according to the change in the shape of host volumes due to different ages and/or aspect 
759 ratios of the human lungs. It should be noted the previous models cannot be applied for generation

760 of bronchial tree of children.

761 - Finding the characteristic properties (i.e. the diameter, length, and direction) of the $762 \quad$ branches independently

763 - Choosing the best direction among the various potential directions based on the proposed $764 \quad$ rules

765 - Using dimensionless parameters and age-dependent terminating criteria

766 - Generation of the airways in parallel

767 It has been shown that if part(s) of lungs are surgically removed, the remaining parts gradually 768 grow in directions that the obstacles have been taken out [53]. Another unique advantage of our

769 method is that the airways do not grow in the host volumes independently to ensure that all

770 interfaces are fed by at least one branch. It also enables the proposed method to simulate the growth

771 of bronchial tree due to surgical removal of the neighbor segment(s) as soon as the growth rate is

772 determined, which requires the clinical studies of the human lungs over the years. It should be

773 noted that the previous models cannot be used for the generation of the structure in which some

774 segments have been surgically removed. Furthermore, the generation of the airways in parallel 775 decreases the computational cost 18 times.

$777 \quad 6$. Conclusion

778 A 3-D structural model of the human conducting airways has been proposed by a stochastic 779 rule-based method that fulfills the anatomical constraints of the human lungs. The airways have 780 propagated into the bronchopulmonary segments whose surfaces have been obtained from the CT 
781 data. The parametric L-system (containing 11 rewriting rules) has been used to generate the

782 structure, in a manner that each module in the rewriting rules represents an airway with its

783 characteristic properties. The diameter, length, and orientation of the airways have been found

784 independently based on an intelligent procedure in contrast to the previous studies that at least two

785 properties were correlated. The morphometric characteristics of the obtained structure have been

786 thoroughly compared against their corresponding experimental data. The results showed that the

787 proposed method outperforms the previous models where it predicted the more accurate

788 dimensions of $\mathrm{TBs}$ and $\mathrm{D} / \mathrm{D}_{\mathrm{p}}$ ratio than the previous asymmetric models. Furthermore, it is more

789 robust and realistic than the previous models. Although the proposed algorithm has been

790 developed based on the healthy subjects, it is capable of generating the abnormal structures if

791 sufficient data is provided for adjustment of the parameters of the proposed method. The realistic

792 structure obtained by the present method can be used in modeling of airflow distribution, aerosol

793 deposition, gas and heat transport. It can be also used to determine the regions of the lung that are

794 damaged by toxic gases or obstructed.

796 Availability of data and materials

797 The CT images used by this study are available in http://image.diku.dk/exact/. All data generated 798 during this study are included in this published article and the additional material "Conducting 799 airways.xlsx" which contains the geometric characteristics of all conducting airways generated by 800 the proposed method.

\section{Competing interests}

802 The authors declare that they have no competing interests.

\section{Funding}

804 This research did not receive any specific grant from funding agencies in the public, commercial, 805 or not-for-profit sectors. 


\section{Authors' contributions}

$807 \mathrm{ZA}$ and RB participated in ideas, development and design of the study, and evolution of the

808 research goals. ZA implemented the computer codes using $\mathrm{C}++$, validated the model results with 809 experimental data, wrote the initial draft, and participated in the editing and revision of final 810 version of the manuscript. RB supervised the research, edited, and revised the manuscript.

\section{Acknowledgements}

814

817 1. Hall JE. Guyton and Hall textbook of medical physiology e-Book: Elsevier Health 818 Sciences; 2010.

819 2. Mitsakou C, Helmis C, Housiadas C. Eulerian modelling of lung deposition with sectional

840 9. Wu D, Tawhai MH, Hoffman EA, Lin C-L. A numerical study of heat and water vapor 841 transfer in MDCT-based human airway models. Annals of biomedical engineering. $842 \quad 2014 ; 42(10): 2117-31$.

843 10. Weibel E. Morphometry of the Human Lung (Academic, New York). Biometrical Journal. 8441963.

845 11. Yeh H-C, Schum G. Models of human lung airways and their application to inhaled particle 846 deposition. Bulletin of mathematical biology. 1980;42(3):461-80.

847 12. Horsfield K, Cumming G. Morphology of the bronchial tree in man. Journal of Applied 848 Physiology. 1968;24(3):373-83. 
13. Horsfield K, Dart G, Olson DE, Filley GF, Cumming G. Models of the human bronchial tree. Journal of applied physiology. 1971;31(2):207-17.

851 14. Koblinger L, Hofmann W. Monte Carlo modeling of aerosol deposition in human lungs.

852 Part I: Simulation of particle transport in a stochastic lung structure. Journal of Aerosol Science. 1990;21(5):661-74.

854 15. Raabe O. Tracheobronchial geometry-human, dog, rat, hamster. Lovelace Foundation for Medical Education and Research Report number LF-53. 1976.

16. Kitaoka H, Takaki R, Suki B. A three-dimensional model of the human airway tree. Journal of Applied Physiology. 1999;87(6):2207-17.

858 17. Murray CD. The physiological principle of minimum work applied to the angle of branching of arteries. The Journal of general physiology. 1926;9(6):835.

860 18. Tawhai MH, Pullan A, Hunter P. Generation of an anatomically based three-dimensional model of the conducting airways. Annals of biomedical engineering. 2000;28(7):793-802. 19. Tawhai MH, Hunter P, Tschirren J, Reinhardt J, McLennan G, Hoffman EA. CT-based geometry analysis and finite element models of the human and ovine bronchial tree. Journal of applied physiology. 2004;97(6):2310-21.

20. Bordas R, Lefevre C, Veeckmans B, Pitt-Francis J, Fetita C, Brightling CE, et al. Development and analysis of patient-based complete conducting airways models. PloS one. 2015;10(12):e0144105.

868 21. Montesantos S, Katz I, Pichelin M, Caillibotte G. The creation and statistical evaluation of 869 a deterministic model of the human bronchial tree from HRCT images. PLOS one. $870 \quad$ 2016;11(12):e0168026.

871 22. Davoodi A, Boozarjomehry RB. Developmental model of an automatic production of the 872 human bronchial tree based on L-system. Computer methods and programs in biomedicine. 873 2016;132:1-10.

874 23. Haefeli-Bleuer B, Weibel ER. Morphometry of the human pulmonary acinus. The 875 Anatomical Record. 1988;220(4):401-14.

876 24. Prusinkiewicz P, Lindenmayer A. The algorithmic beauty of plants: Springer Science \& 877 Business Media; 2012.

878 25. Lindenmayer A. Mathematical models for cellular interactions in development I. Filaments with one-sided inputs. Journal of theoretical biology. 1968;18(3):280-99.

26. Ijiri T, Owada S, Igarashi T, editors. The sketch 1-system: Global control of tree modeling using free-form strokes. International Symposium on Smart Graphics; 2006: Springer.

27. Prusinkiewicz P. Modeling plant growth and development. Current opinion in plant biology. 2004;7(1):79-83.

28. Zamir M. Arterial branching within the confines of fractal L-system formalism. The Journal of general physiology. 2001;118(3):267-76.

29. Weibel ER, Cournand AF, Richards DW. Morphometry of the human lung: Springer; 1963.

30. Nikiforov AI, Schlesinger RB. Morphometric variability of the human upper bronchial tree. Respiration physiology. 1985;59(3):289-99.

31. Phalen RF, Oldham MJ, Beaucage CB, Crocker TT, Mortensen J. Postnatal enlargement of human tracheobronchial airways and implications for particle deposition. The Anatomical Record. 1985;212(4):368-80.

32. Mauroy B, Filoche M, Weibel E, Sapoval B. An optimal bronchial tree may be dangerous. Nature. 2004;427(6975):633.

33. Phillips C, Kaye S. Diameter-based analysis of the branching geometry of four mammalian bronchial trees. Respiration physiology. 1995;102(2-3):303-16. 
34. Thurlbeck A, Horsfield K. Branching angles in the bronchial tree related to order of branching. Respiration physiology. 1980;41(2):173-81.

35. Horsfield K, Thurlbeck A. Volume of the conducting airways calculated from morphometric parameters. Bulletin of mathematical biology. 1981;43(1):101-9.

36. Horsfield K, Gordon W, Kemp W, Phillips S. Growth of the bronchial tree in man. Thorax. 1987;42(5):383-8.

37. Extraction of Airways from CT [Internet]. 2009. Available from: http://image.diku.dk/exact/.

38. Lobe and Lung analysis 2011 [Available from: https://lola11.grand-challenge.org/.

39. Schreider JP, Raabe OG. Structure of the human respiratory acinus. American Journal of Anatomy. 1981;162(3):221-32.

40. Litzlbauer HD, Korbel K, Kline TL, Jorgensen SM, Eaker DR, Bohle RM, et al. Synchrotron-Based Micro-CT Imaging of the Human Lung Acinus. The Anatomical Record. 2010;293(9):1607-14.

41. Horsfield K, Cumming G. Angles of branching and diameters of branches in the human bronchial tree. The Bulletin of mathematical biophysics. 1967;29(2):245-59.

42. Uylings H. Optimization of diameters and bifurcation angles in lung and vascular tree structures. Bulletin of mathematical biology. 1977;39(5):509-20.

43. Möller T, Trumbore B, editors. Fast, minimum storage ray/triangle intersection. ACM SIGGRAPH 2005 Courses; 2005: ACM.

44. Havel J, Herout A. Yet faster ray-triangle intersection (using SSE4). IEEE Transactions on Visualization and Computer Graphics. 2010;16(3):434-8.

45. Nedjah N, de Macedo Mourelle L. Fuzzy systems engineering: theory and practice: Springer Science \& Business Media; 2005.

46. Ménache M, Hofmann W, Ashgarian B, Miller F. Airway geometry models of children's lungs for use in dosimetry modeling. Inhalation toxicology. 2008;20(2):101-26.

47. Phalen R, Yeh H, Schum G, Raabe O. Application of an idealized model to morphometry of the mammalian tracheobronchial tree. The Anatomical Record. 1978;190(2):167-76.

48. Horsfield K, Relea FG, Gumming G. Diameter, length and branching ratios in the bronchial tree. Respiration physiology. 1976;26(3):351-6.

49. Litzlbauer HD, Korbel K, Kline TL, Jorgensen SM, Eaker DR, Bohle RM, et al. Synchrotron-Based Micro-CT Imaging of the Human Lung Acinus. The Anatomical Record: Advances in Integrative Anatomy and Evolutionary Biology. 2010;293(9):1607-14.

50. Soong T, Nicolaides P, Yu C, Soong S. A statistical description of the human tracheobronchial tree geometry. Respiration physiology. 1979;37(2):161-72.

51. Ismail $\mathrm{M}$, Comerford $\mathrm{A}$, Wall $\mathrm{W}$. Coupled and reduced dimensional modeling of respiratory mechanics during spontaneous breathing. International journal for numerical methods in biomedical engineering. 2013;29(11):1285-305.

52. Nascimento WV, Cassiani RA, Dantas RO. Gender effect on oral volume capacity. Dysphagia. 2012;27(3):384-9.

53. Butler JP, Loring SH, Patz S, Tsuda A, Yablonskiy DA, Mentzer SJ. Evidence for adult lung growth in humans. New England Journal of Medicine. 2012;367(3):244-7. 